\title{
Stress Intensity Factor Solutions for Welds in Lap-Shear Specimens of Dissimilar Sheet Materials with and without Kinked Cracks
}

\author{
Teresa J. Rinker ${ }^{\mathrm{a}, 1}$ and Jwo Pan ${ }^{\mathrm{b}}$ \\ ${ }^{a}$ Manufacturing Systems Research Laboratory \\ GM Global R\&D \\ 30500 Mound Road, Warren, MI 48092-2031, USA \\ ${ }^{\mathrm{b}}$ Mechanical Engineering \\ University of Michigan \\ Ann Arbor, MI 48109, USA
}

March 20, 2017

\begin{abstract}
Global stress intensity factor solutions for welds with various widths in lap-shear specimens of magnesium alloy AZ31 and high-strength low-alloy steel sheets are developed using finite element analyses. Results are compared to and validated with the analytical solutions based on the beam bending theory and semi-infinite planes with connection. Analyses are also conducted using modified elastic constants with a bimaterial constant of zero for specimens with small kinked cracks. Results indicate that the stress intensity factor solutions for a vanishing kink length can be approximated by the available analytical solutions for the zero bimaterial constant for fatigue cracks growth models. The results of the global stress intensity factor solutions and the local stress intensity factor solutions for kinked cracks with the experimentally observed kink angle as functions of the kink length can be adopted for fatigue life estimations of ultrasonic welds in lap-shear specimens of magnesium and steel sheets.
\end{abstract}

\footnotetext{
${ }^{1}$ Corresponding author. Tel.:+1-248-794-4334; fax:+1-586-986-3091

E-mail address: teresa.rinker@gm.com (T. J. Rinker)
} 
Keywords: ultrasonic weld, dissimilar welds, lap-shear specimen, stress intensity factor, kinked crack

\section{Nomenclature}

\begin{tabular}{|c|c|}
\hline$a$ & kinked crack length \\
\hline$a / t$ & ratio of the kink length to the sheet thickness \\
\hline$b$ & reduced specimen width \\
\hline$c, d$ & complex-valued functions of $\varphi, \alpha$ and $\beta$ \\
\hline$E$ & Young's modulus \\
\hline$E^{\prime}$ & effective Young's modulus under plane strain conditions \\
\hline$f_{I}, f_{I I}, f_{e}$ & dimensionless geometric function \\
\hline$F$ & resultant force \\
\hline$g$ & indentation width \\
\hline$G$ & shear modulus \\
\hline$k_{I}, k_{I I}$ & local stress intensity factors \\
\hline$k_{e}$ & effective local stress intensity factor \\
\hline$K_{e}$ & effective global stress intensity factor \\
\hline $\mathbf{K}=K_{1}+i K_{2}$ & complex stress intensity factor for an interface crack \\
\hline$L$ & specimen length \\
\hline$r$ & radius of reduced area \\
\hline$t$ & sheet thickness \\
\hline$V$ & specimen overlap length \\
\hline
\end{tabular}




$\begin{array}{ll}W & \text { specimen width in the grip section } \\ w & \text { ultrasonic weld width } \\ w / t & \text { ratio of the weld width to the sheet thickness } \\ \alpha, \beta & \text { Dundurs' parameters } \\ \delta=t_{u} / t_{l} & \text { thickness ratio } \\ \varepsilon & \text { bimaterial constant } \\ \eta=E^{\prime}{ }_{u} / E_{l}{ }_{l} & \text { modulus ratio } \\ v & \text { Poisson's ratio } \\ \sigma & \text { normal structural stress } \\ \sigma^{*} & \text { normal structural stress to satisfy the equilibrium and the continuity of the } \\ & \end{array}$

Superscripts

A stress intensity factors from Abaqus which are expressed in the form of Equation (35)

E stress intensity factors of Erdogan [25] which are expressed in the form of Equation (21)

Subscripts

E stress intensity factors of Erdogan [25] which are transformed into the form of Equation (1)

$u, l \quad$ upper and lower sheets of weld 
$i, o \quad$ inner and outer surfaces of the sheets

TPI stress intensity factors from Tada et al. [29]

\section{Introduction}

The advantage of solid state joining methods such as ultrasonic welding and friction stir welding is that melting is either avoided or minimized. Both solid state joining methods can be used to join dissimilar metals such as magnesium and steel sheets. Resistance spot welding of magnesium alloys could produce hot cracking [1], while ultrasonic spot welding can be used to successfully join magnesium alloys [2]. Researchers have been using ultrasonic welding or friction stir welding to join both similar and dissimilar materials. Ultrasonic spot welds between aluminum sheets have been made by many researchers, including Hetrick et al. [3], Jahn et al. [4], and Bakavos and Prangnell [5]. Friction stir spot welds between magnesium sheets have been made, for example, see Jordan et al. [6]. Dissimilar welds have been produced most commonly between aluminum and steel sheets by researchers such as Gendo et al. [7], Liyanage et al. [8], and Watanabe et al. [9]. Dissimilar welds were also produced between magnesium and steel sheets by Santella et al. [10] Jana and Hovanski [11] and Uematsu et al. [12].

Dissimilar welds need to undergo fatigue testing as components with welded lap joints are frequently exposed to cyclic loading conditions. In lap joints, fatigue cracks tend to initiate from the pre-existing crack tips at the edge of a weld. Some researchers including Patel et al. [13] and Shen et al.[14] have investigated the fatigue behavior of solid state welds. This is an important and necessary step in order to develop life prediction and design tools for product development.

In this study, ultrasonic spot welded lap-shear specimens of magnesium alloy AZ31 and high strength low alloy (HSLA) steel sheets were machined into a dog-bone shape. The experimental 
results based on these dog-bone specimens can be analyzed by using two-dimensional plane strain finite element analyses and available analytical stress intensity factor solutions. Additionally, certain ultrasonic welders are designed to produce seam welds such as those studied by Ueoka and Tsujino [15]. The results of this research can be directly applicable to dissimilar seam welds.

Fatigue crack growth models based on fracture mechanics rely on stress intensity factor solutions for the welds in lap-shear specimens. Many researchers developed stress intensity factor solutions for similar and dissimilar welds in lap-shear specimens. For example, Sripichai et al. [16] developed stress intensity factor solutions for similar laser welds in lap-shear specimens based on the beam bending theory. Zhang $[17,18]$ developed stress intensity factor solutions for dissimilar welds in lap-shear specimens of different materials based on the $J$ integral and the work of Suo and Hutchinson [19].

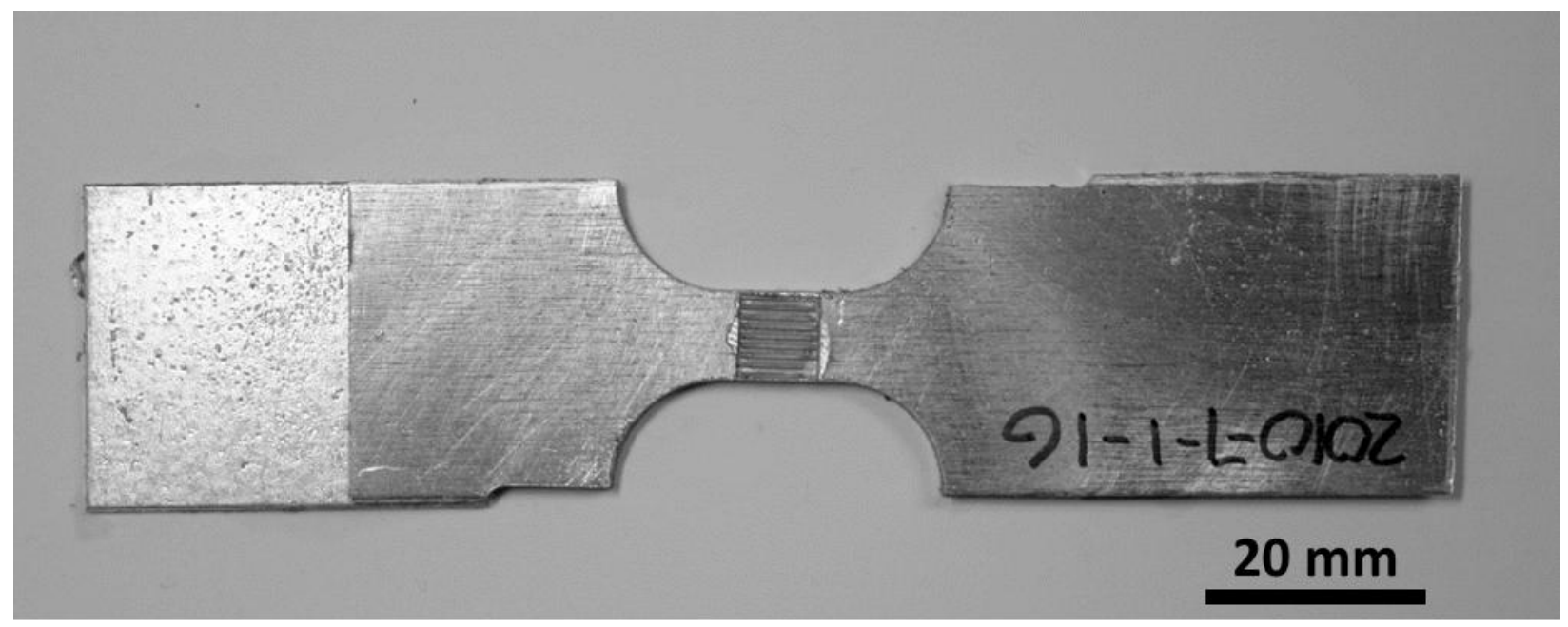

(a) 


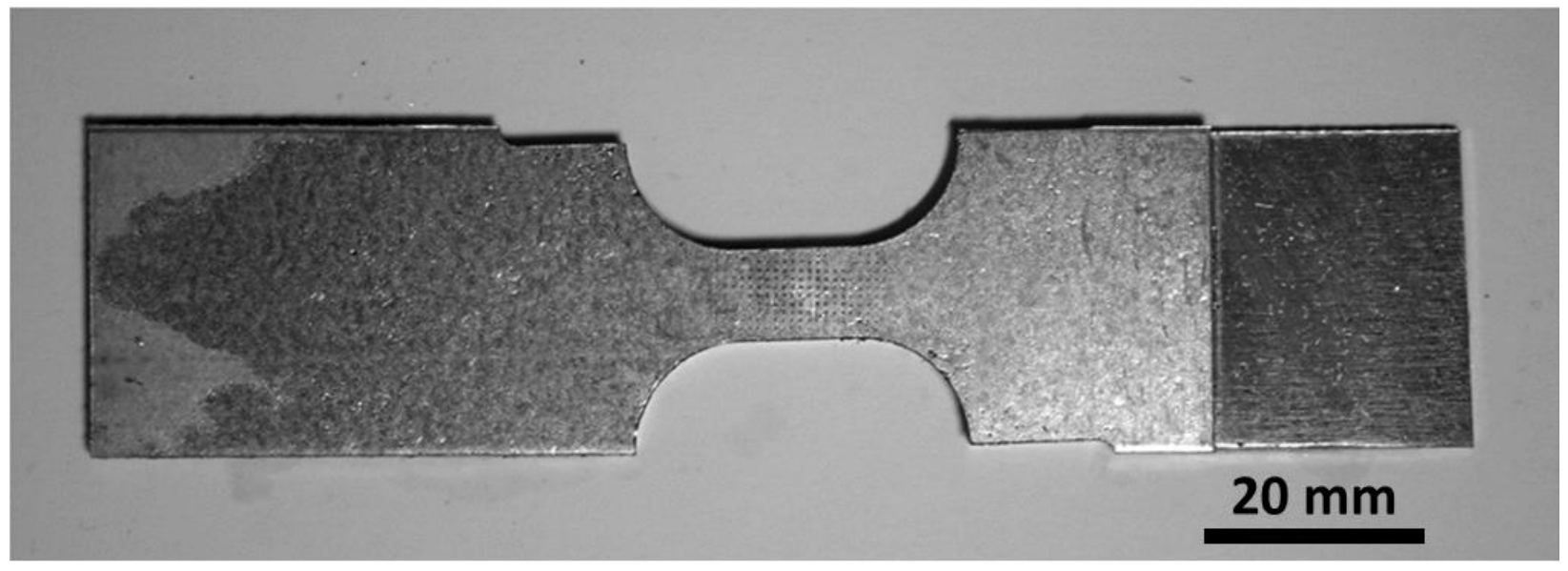

(b)

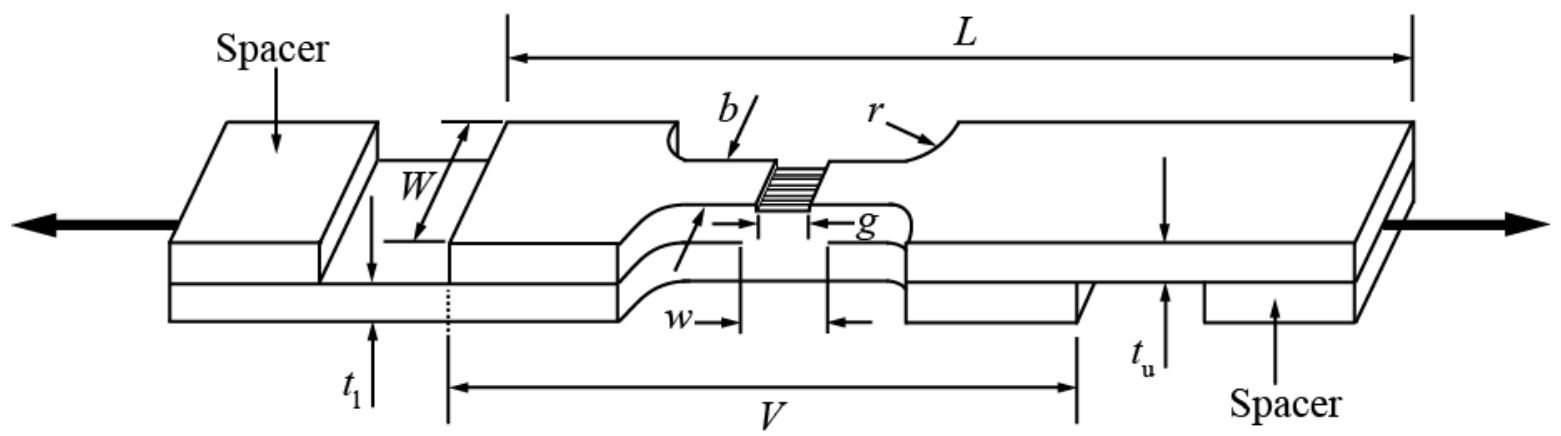

(c)

Figure 1. (a) A top view and (b) a bottom view of an ultrasonic welded lap-shear specimen which has been machined into a dog-bone shape. (c) A schematic of a lap-shear specimen. The applied force $F$ is shown as the bold arrows.

Figures 1(a) and 1(b) show the top and bottom views of an ultrasonic spot welded lap-shear specimen after being machined into a dog-bone shape. Figure 1(c) shows a schematic of a lapshear specimen with the loading direction represented by bold arrows. As shown in the figure, the specimen has a width $W$ and overall length $L$ for the upper and lower sheets. The specimen has the thickness $t_{u}$ for the upper sheet and $t_{l}$ for the lower sheet. The specimen has an overlap length $V$. The reduced section in the middle of the specimen has a reduced width $b$ and a transition radius $r$. The width of the sontrode tip indentation on the top surface of the upper 
sheet is denoted by $g$. The weld width $w$ of the ultrasonic weld is defined as the distance between the two pre-existing crack tips as shown in Figure 1(c). Experimentally, the ultrasonic weld zone can be observed from failed specimens and is larger than the sontrode tip indentation on the top surface. For analytical and computational analyses presented in this paper, the sonotrode tip indentation will not be considered. The specimen has two spacers to align the applied force $F$, shown with bold arrows, along the weld interface. The materials in the weld zone are assumed to have the same elastic properties as the two base metals. The dimensions of the specimens are $W=30 \mathrm{~mm}, L=100 \mathrm{~mm}, b=8 \mathrm{~mm}, V=75 \mathrm{~mm}, w=8.78 \mathrm{~mm}, g=7$ $\mathrm{mm}$ and $r=10 \mathrm{~mm}$. The thickness $t_{u}$ for the upper magnesium sheet is $1.58 \mathrm{~mm}$ and the thickness $t_{l}$ for the lower steel sheet is $1.5 \mathrm{~mm}$. Note that the upper and lower sheets have nearly the same thickness. Specimens with similar shapes were adopted by a number of researchers for the study of laser welded joints, for example, see Lee et al. [20], Asim et al. [21], Anand et al. [22], and Asim et al. [23]. The geometry of the lap-shear specimen investigated here varies slightly from the idealized specimen used in the finite element models. In the idealized model, the thickness of the magnesium sheet is taken to be $1.50 \mathrm{~mm}$ to provide a lapshear specimen of equal thickness.

Figure 2 shows a micrograph of the cross section of a failed ultrasonic weld in a lap-shear specimen at the fatigue life of $1.7 \times 10^{3}$ cycles under the load range of $1.70 \mathrm{kN}$. With regard to the figure, load is applied to the right on the right edge of the upper sheet and to the left on the left edge of the lower sheet as shown schematically with the arrows. As shown in Figure 2, a fatigue crack is initiated near the main crack tip on the right side of the weld. The fatigue crack can be considered as a kinked crack with respect to the right main crack tip. All of the observed partially and fully failed ultrasonic welds displayed kink angles close to $90^{\circ}$. 


\section{Mg alloy}

\section{Steel}

\section{$1 \mathrm{~mm}$}

Figure 2. An optical micrograph of a failed weld at the fatigue life of $1.7 \times 10^{3}$ cycles under a load range of $1.70 \mathrm{kN}$.

As shown in Figure 2, the weld failed by the kinked crack emanating from the right main crack. In order to determine the fatigue life of the weld based on a kinked fatigue crack growth model, stress intensity factor solutions for kinked cracks with various lengths are needed. Therefore, in this investigation, the local stress intensity factors for kinked cracks will be obtained by finite element analyses. He and Hutchinson [24] developed local stress intensity factor solutions for a small kinked crack growing from the bimaterial interface. However, for a material combination with a nonzero bimaterial constant, the local stress intensity factor solutions vary with the distance to the tip due to the stress oscillation which occurs at distances extremely close to the main crack tip. Therefore, finite element analyses are used to investigate the asymptotic crack-tip fields near main cracks and kinked cracks with very small kink lengths for a zero and nonzero bimaterial constant.

In this paper, the global stress intensity factor solutions for dissimilar welds in lap-shear specimens will first be investigated for various weld widths. Two-dimensional finite element models are developed to obtain the stress intensity factor solutions for selected weld widths 
under plane strain conditions. The computational stress intensity factor solutions are compared with the global stress intensity factor solutions obtained from the beam bending theory and those found from the elasticity theory for two dissimilar semi-infinite planes with a connection as derived by Erdogan [25]. Next, the crack-tip stress solutions are obtained from the finite element analyses based on the two material models for a zero and nonzero bimaterial constant in order to explore the use of the asymptotic solutions of He and Hutchinson [24] for the vanishing kink length for fatigue life estimations. Then, finite element models for three weld widths are developed to obtain the local stress intensity factor solutions for kinked cracks with various lengths. The global stress intensity factor solutions and local stress intensity factor solutions for vanishing and finite kinked cracks can then be used to estimate the fatigue lives of the dissimilar welds in lap-shear specimens of magnesium and steel sheets. The corresponding experimental results and fatigue life estimations will be reported in the future.

\section{Analytical global stress intensity factor solutions for main cracks}

The asymptotic in-plane stress field around an interface crack tip is an oscillatory field that

can be characterized by a complex stress intensity factor $\mathbf{K}\left(=K_{1}+i K_{2}, i=\sqrt{-1}\right)$ (Rice and Sih [26] and Rice [27]). The stresses $\sigma_{y}$ and $\tau_{x y}$ at a small distance $r$ ahead of the interface crack tip are characterized by $\mathbf{K}$ as

$$
\sigma_{y}+i \tau_{x y}=\frac{K_{1}+i K_{2}}{\sqrt{2 \pi r}}\left(\frac{r}{t}\right)^{i \varepsilon}
$$

Here, the bimaterial constant $\varepsilon$ is defined as

$$
\varepsilon=\frac{1}{2 \pi} \ln \frac{\kappa_{u} / G_{u}+1 / G_{l}}{\kappa_{l} / G_{l}+1 / G_{u}}
$$


where $G_{u}$ represents the shear modulus of the upper sheet and $G_{l}$ represents the shear modulus of the lower sheet. Here, $\kappa_{u}$ and $\kappa_{l}$ for the upper and lower sheets, respectively, are defined as

$$
\kappa_{u}=3-4 v_{u}
$$

and

$$
\kappa_{l}=3-4 v_{l}
$$

under plane strain conditions. Note that $v_{u}$ and $v_{l}$ represent the Poisson's ratios of the upper and lower sheets, respectively.

In Equation (1), $t$ represents a characteristic length [27, 28]. In this investigation, $t$ represents the upper sheet thickness $t_{u}$ which is the smaller value of the thicknesses of the two sheets bonded together as in Suo and Hutchinson [19] and Zhang [28]. It should be noted that when the two materials are identical, $\varepsilon=0$. With identical materials, $K_{1}$ and $K_{2}$ in Equation (1) for the interface crack become the conventional stress intensity factors $K_{I}$ and $K_{I I}$, respectively.

The reduced section in the middle of the lap-shear specimen shown in Figure 1 can be modeled as two sheets with connection under lap-shear loading conditions. Figure 3(a) shows a schematic for two sheets with connection. The width of the connection is $w$. The two beams are subject to a lap-shear load $F / b$.

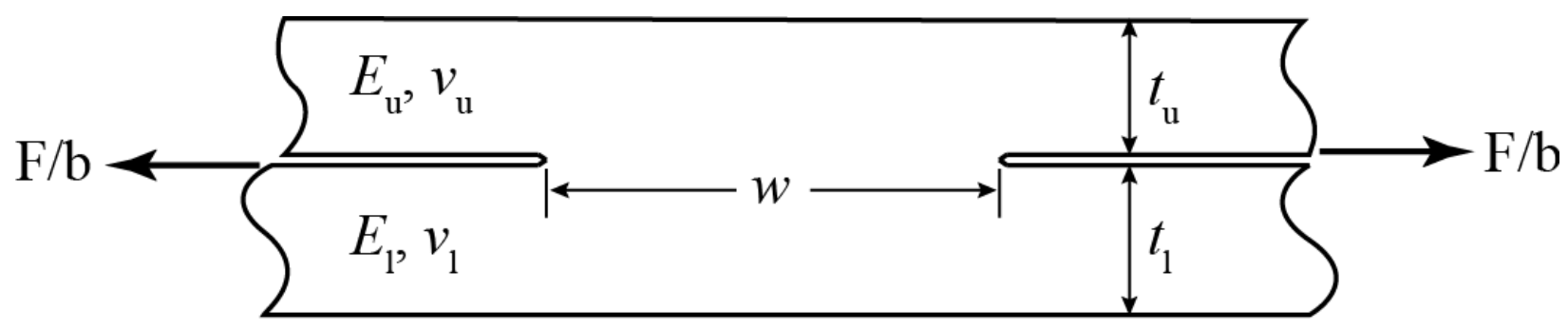

(a) 


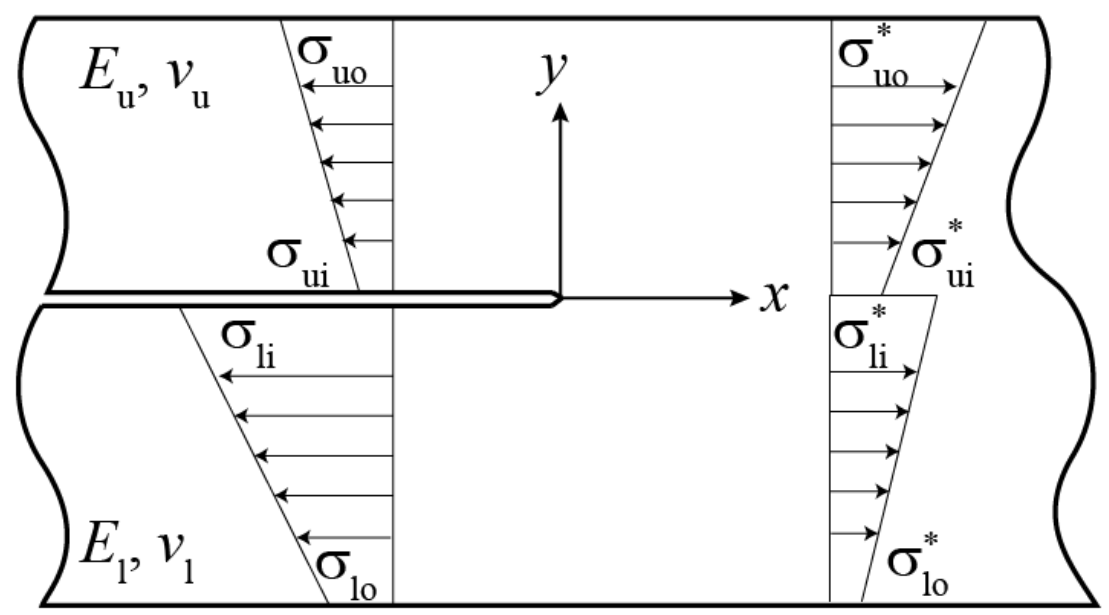

(b)

Figure 3. (a) A schematic of the weld with the lap-shear load. (b) A schematic of the left crack tip showing the normal stresses $\sigma_{u i}, \sigma_{u o}, \sigma_{l i}$ and $\sigma_{l o}$ at the inner $(i)$ and outer $(o)$ surfaces of the upper $(u)$ and lower $(l)$ strips, respectively.

The global stress intensity factor solutions, $K_{1}$ and $K_{2}$, derived by Zhang [28] are

$$
\begin{aligned}
K_{1} & =\frac{\cosh (\pi \varepsilon) \sqrt{t_{u}}}{2 \sqrt{3(1+\eta)\left(1+4 \eta \delta+6 \eta \delta^{2}+3 \eta \delta^{3}\right)\left(1+\tan ^{2} \omega\right)}} \times \\
& \left\{\left[\frac{\left(1+4 \eta \delta+9 \eta \delta^{2}+6 \eta \delta^{3}\right) \tan \omega}{\sqrt{1+2 \eta \delta\left(2+3 \delta+2 \delta^{2}\right)+\eta^{2} \delta^{4}}}-\sqrt{3}\right] \sigma_{u i}\right. \\
& -\left[\frac{\left(1+4 \eta \delta+3 \eta \delta^{2}\right) \tan \omega}{\sqrt{1+2 \eta \delta\left(2+3 \delta+2 \delta^{2}\right)+\eta^{2} \delta^{4}}}+\sqrt{3}\right] \sigma_{u o} \\
& +\eta\left[\frac{\delta\left(1-2 \eta \delta-3 \eta \delta^{2}\right) \tan \omega}{\sqrt{1+2 \eta \delta\left(2+3 \delta+2 \delta^{2}\right)+\eta^{2} \delta^{4}}}+\sqrt{3}(2+\delta)\right] \sigma_{l i} \\
& \left.-\eta \delta\left[\frac{\left(1+4 \eta \delta+3 \eta \delta^{2}\right) \tan \omega}{\sqrt{1+2 \eta \delta\left(2+3 \delta+2 \delta^{2}\right)+\eta^{2} \delta^{4}}}+\sqrt{3}\right] \sigma_{l o}\right\} \\
K_{2} & =\frac{\cosh (\pi \varepsilon) \sqrt{t_{u}}}{2 \sqrt{3(1+\eta)\left(1+4 \eta \delta+6 \eta \delta^{2}+3 \eta \delta^{3}\right)\left(1+\tan ^{2} \omega\right)}} \times \\
& \left\{\left[\frac{\left(1+4 \eta \delta+9 \eta \delta^{2}+6 \eta \delta^{3}\right)}{\sqrt{1+2 \eta \delta\left(2+3 \delta+2 \delta^{2}\right)+\eta^{2} \delta^{4}}}+\sqrt{3}{\tan \omega] \sigma_{u i}}\right.\right.
\end{aligned}
$$




$$
\begin{aligned}
& -\left[\frac{\left(1+4 \eta \delta+3 \eta \delta^{2}\right)}{\sqrt{1+2 \eta \delta\left(2+3 \delta+2 \delta^{2}\right)+\eta^{2} \delta^{4}}}-\sqrt{3} \tan \omega\right] \sigma_{u o} \\
& +\eta\left[\frac{\delta\left(1-2 \eta \delta-3 \eta \delta^{2}\right)}{\sqrt{1+2 \eta \delta\left(2+3 \delta+2 \delta^{2}\right)+\eta^{2} \delta^{4}}}-\sqrt{3}(2+\delta) \tan \omega\right] \sigma_{l i} \\
& -\eta \delta\left[\frac{\left(1+4 \eta \delta+3 \eta \delta^{2}\right)}{\sqrt{1+2 \eta \delta\left(2+3 \delta+2 \delta^{2}\right)+\eta^{2} \delta^{4}}}-\sqrt{3} \tan \omega\right] \sigma_{l o}
\end{aligned}
$$

where the modulus ratio $\eta$ is defined as

$$
\eta=E_{u}^{\prime} / E_{l}^{\prime}
$$

Here,

$$
E_{u}^{\prime}=E_{u} /\left(1-v_{u}^{2}\right)
$$

and

$$
E_{l}^{\prime}=E_{l} /\left(1-v_{l}^{2}\right)
$$

where $E_{u}$ and $E_{l}$ represent the Young's moduli of the upper and lower sheets, respectively.

The thickness ratio $\delta$ is defined as

$$
\delta=t_{u} / t_{l}
$$

where $t_{u}$ and $t_{l}$ are the upper and lower sheet thicknesses, respectively. Note that the expressions shown in Equations (5) and (6) are for welds joining sheets with $\delta \leq 1$. For welds joining sheets with $\delta>1$, one should rotate the strip model by an angle of $180^{\circ}$ to represent the same physical system but with $\delta<1$.

The values of the angular quantity $\omega$ in Equations (5) and (6) can be found in Suo and Hutchinson [19]. The angular quantity $\omega$ is a function of the thickness ratio $\delta$ and the Dundurs' parameters $\alpha$ and $\beta$ which are defined as

$$
\alpha=\frac{\left(\kappa_{l}+1\right) G_{u}-\left(\kappa_{u}+1\right) G_{l}}{\left(\kappa_{l}+1\right) G_{u}+\left(\kappa_{u}+1\right) G_{l}}
$$




$$
\beta=\frac{\left(\kappa_{l}-1\right) G_{u}-\left(\kappa_{u}-1\right) G_{l}}{\left(\kappa_{l}+1\right) G_{u}+\left(\kappa_{u}+1\right) G_{l}}
$$

Recall that the lap-shear specimen is loaded with a force per unit width, $F / b$, applied to the lower left and upper right sheets along the interface as shown in Figure 3(a). Figure 3(b) shows the left part of the strip model near the crack tip with linearly distributed structural stresses based on the classical Kirchhoff plate theory based on the work of Zhang [28]. As shown in Figure 3(b), $\sigma_{u i}, \sigma_{u o}, \sigma_{l i}$ and $\sigma_{l o}$ represent the normal stresses at the inner $(i)$ and outer $(o)$ surfaces of the upper $(u)$ and lower $(l)$ strips, respectively. Note also that the normal stresses $\sigma_{u i}^{*}, \sigma_{u o}^{*}, \sigma_{l i}^{*}$ and $\sigma_{l o}^{*}$ can be derived from the normal structural stresses $\sigma_{u i}, \sigma_{u o}, \sigma_{l i}$ and $\sigma_{l o}$ based on the equilibrium equations and the continuity conditions of the strain and the strain gradient along the bond line. The normal structural stresses $\sigma_{u i}, \sigma_{u o}, \sigma_{l i}$ and $\sigma_{l o}$ which are marked in Figure 3(b) for the left crack tip are

$$
\begin{aligned}
& \sigma_{u o, l e f t}=0 \\
& \sigma_{u i, l e f t}=0 \\
& \sigma_{l i, l e f t}=\frac{4 F}{t_{l} b} \\
& \sigma_{l o, l e f t}=-\frac{2 F}{t_{l} b}
\end{aligned}
$$

The normal structural stresses $\sigma_{u i}, \sigma_{u o}, \sigma_{l i}$ and $\sigma_{l o}$ for the right crack tip are

$$
\begin{gathered}
\sigma_{u o, \text { right }}=-\frac{2 F}{t_{u} b} \\
\sigma_{u i, r i g h t}=\frac{4 F}{t_{u} b}
\end{gathered}
$$




$$
\begin{aligned}
& \sigma_{l i, \text { right }}=0 \\
& \sigma_{l o, \text { right }}=0
\end{aligned}
$$

The values of the analytical global stress intensity factors, $K_{1}$ and $K_{2}$, for the left and right tips of the weld can be obtained from Equations (5) and (6) based on Equations (13)-(20).

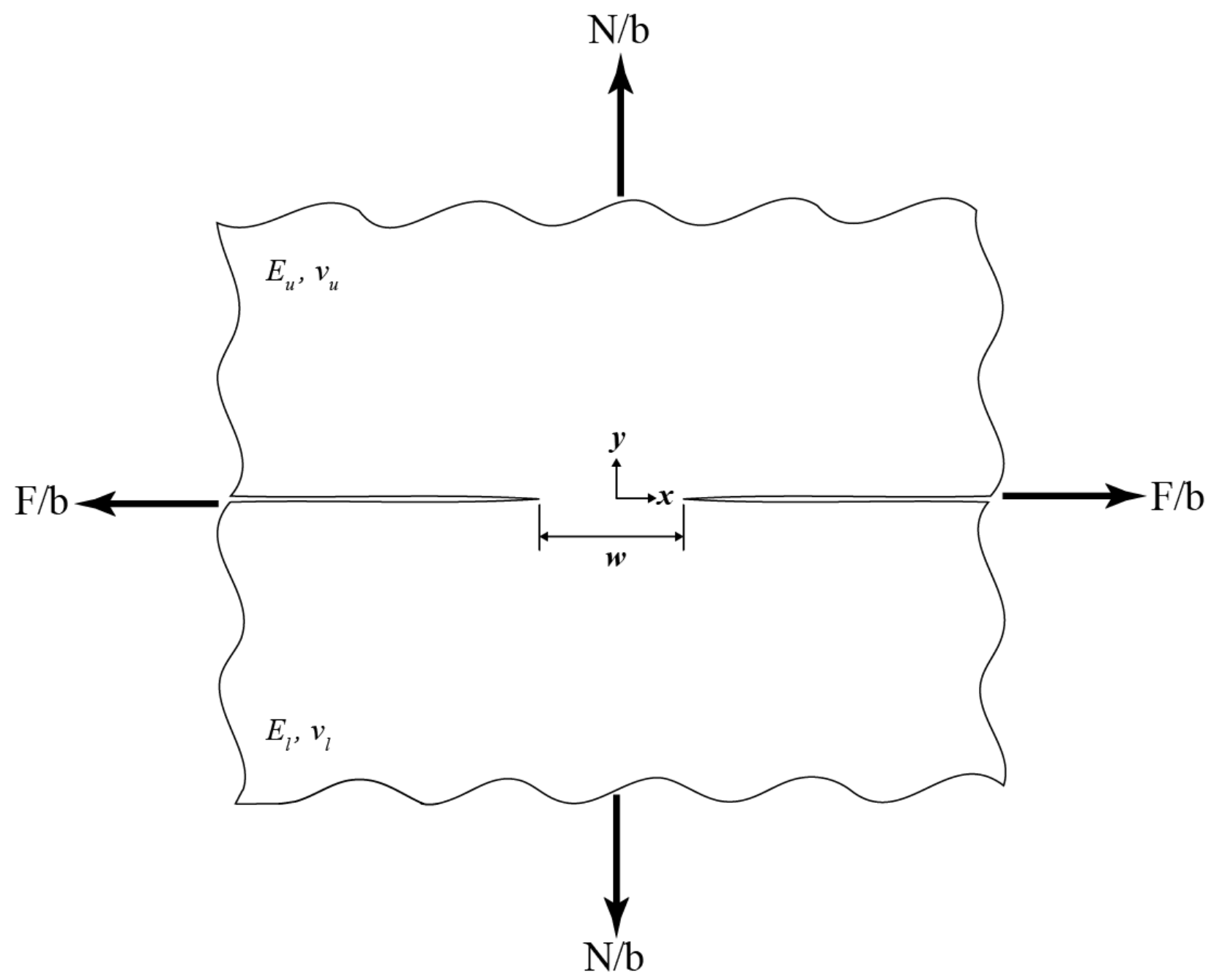

Figure 4. A schematic diagram of two half planes of dissimilar materials with connection of the length $w$. The Cartesian $x-y$ coordinate system is shown. The shear forces per unit width, $F / b$, are applied along the $x$ axis at $x=+\infty$ and $-\infty$ of the upper and lower planes, respectively. The normal forces per unit width, $N / b$, are applied along the $y$ axis at $y=+\infty$ and $-\infty$ of the upper and lower planes, respectively. 
When the weld width $w$ becomes small compared to the upper thickness $t_{u}$, the stress intensity factor $K_{1}$ and $K_{2}$ solutions should approach to those for two dissimilar semi-infinite planes with connection under shear loading conditions as presented by Erdogan [25]. Figure 4 shows a schematic diagram of two dissimilar half planes with connection of the length $w$ under remote shear and normal loads. The Cartesian $x-y$ coordinate system is also shown. The shear forces per unit width, $F / b$, are applied along the $x$ axis at $x=+\infty$ and $x=-\infty$ of the upper and lower planes, respectively. The normal forces per unit width, $N / b$, are applied along the $y-$ axis at $y=+\infty$ and $y=-\infty$ of the two planes, respectively. The stress intensity factor $K_{1}^{E}$ and $K_{2}^{E}$ solutions of Erdogan [25] can be expressed in the form

$$
\sigma_{y}+i \tau_{x y}=\frac{K_{1}^{E}+i K_{2}^{E}}{\sqrt{2 \pi r}}\left(\frac{r}{w}\right)^{i \varepsilon}
$$

where $K_{1}^{E}$ and $K_{2}^{E}$ are

$$
\begin{aligned}
& K_{1}^{E}=\frac{\sqrt{2}}{\sqrt{\pi w}} \frac{N}{b} \cosh (\pi \varepsilon) \\
& K_{2}^{E}=\frac{\sqrt{2}}{\sqrt{\pi w}} \frac{F}{b} \cosh (\pi \varepsilon)
\end{aligned}
$$

For homogenous materials, $\varepsilon=0$. The stress intensity factor solutions in Equations (22) and (23) become $K_{\text {I,TPI }}$ and $K_{\text {II,TPI }}$, [16], based on the Westergaard stress function as in Tada et al. [29] as

$$
K_{\mathrm{I}, \mathrm{TPI}}=\frac{\sqrt{2} N}{b \sqrt{\pi w}}
$$

and 


$$
K_{\mathrm{II}, \mathrm{TPI}}=\frac{\sqrt{2} F}{b \sqrt{\pi w}}
$$

It should be noted that in the original form of the Erdogan solutions, the radius is normalized by the connection length $w$ as can be observed in Equation (21). The connection length $w$ is the only relevant length scale in the original problem of two dissimilar half planes with connection. However, for the application of dissimilar welds, the sheet thickness $t$ in Equation (1) can be used for normalization. To find the conversion, Equation (1) and Equation (21) are set equal as

$$
\left(K_{1}+i K_{2}\right)\left(\frac{r}{t}\right)^{i \varepsilon}=\left(K_{1}^{E}+i K_{2}^{E}\right)\left(\frac{r}{w}\right)^{i \varepsilon}
$$

Simplification yields

$$
K_{1}+i K_{2}=\left(K_{1}^{E}+i K_{2}^{E}\right)\left(\frac{t}{w}\right)^{i \varepsilon}
$$

Using Euler's formula, the equation is rewritten as

$$
K_{1}+i K_{2}=\left(K_{1}^{E}+i K_{2}^{E}\right)\left(\cos \left(\varepsilon \ln \frac{t}{w}\right)+i \sin \left(\varepsilon \ln \frac{t}{w}\right)\right)
$$

Finally, the stress intensity factor solutions of Erdogan [25] are transformed into the form of Equation (1) as

$$
\begin{aligned}
& K_{1}=K_{1}^{E} \cos \left(\varepsilon \ln \frac{t}{w}\right)-K_{2}^{E} \sin \left(\varepsilon \ln \frac{t}{w}\right) \\
& K_{2}=K_{1}^{E} \sin \left(\varepsilon \ln \frac{t}{w}\right)+K_{2}^{E} \cos \left(\varepsilon \ln \frac{t}{w}\right)
\end{aligned}
$$

Substituting Equations (22) and (23) into (29) and (30) gives

$$
K_{1, E}=\frac{\sqrt{2}}{b \sqrt{\pi w}}\left(N \cos \left(\varepsilon \ln \frac{t}{w}\right)-F \sin \left(\varepsilon \ln \frac{t}{w}\right)\right) \cosh (\pi \varepsilon)
$$




$$
K_{2, E}=\frac{\sqrt{2}}{b \sqrt{\pi w}}\left(N \sin \left(\varepsilon \ln \frac{t}{w}\right)+F \cos \left(\varepsilon \ln \frac{t}{w}\right)\right) \cosh (\pi \varepsilon)
$$

For lap-shear loading, $N=0$. Therefore, the stress intensity factor solutions can be simplified to

$$
\begin{aligned}
& K_{1, E}=\frac{\sqrt{2}}{b \sqrt{\pi w}}\left(-F \sin \left(\varepsilon \ln \frac{t}{w}\right)\right) \cosh (\pi \varepsilon) \\
& K_{2, E}=\frac{\sqrt{2}}{b \sqrt{\pi w}}\left(F \cos \left(\varepsilon \ln \frac{t}{w}\right)\right) \cosh (\pi \varepsilon)
\end{aligned}
$$

\section{Computational global stress intensity factor solutions for main cracks}

Global stress intensity factor solutions as functions of weld width were obtained using finite element analyses. The global stress intensity factor solutions from finite element were then compared with the two sets of analytical solutions. The two-dimensional plane strain finite elemental model of the lap-shear specimen with the boundary and loading conditions is shown in Figure 5(a). Note that the upper and lower sheets of the lap-shear specimens used in the experiments have nearly the same thickness. Therefore, the upper and lower sheets in the finite element models in this investigation are idealized with the same thickness $t\left(=t_{u}=t_{l}\right)$. The specimen is modeled in a two-dimensional plane strain finite element model with length $L=100$ mm, overlap length $V=75 \mathrm{~mm}$, and sheet thickness $t=1.5 \mathrm{~mm}$. The width $b=8 \mathrm{~mm}$ of the reduced section in the middle of the specimen, as shown in Figure 1(c), is used in the model. The weld width $w$ is modeled computationally for multiple widths. The finite element model has boundary conditions of fixed displacement and a concentrated force per unit width, $F / b$, applied in the $+x$ direction at the middle surface for left and right edges respectively. 


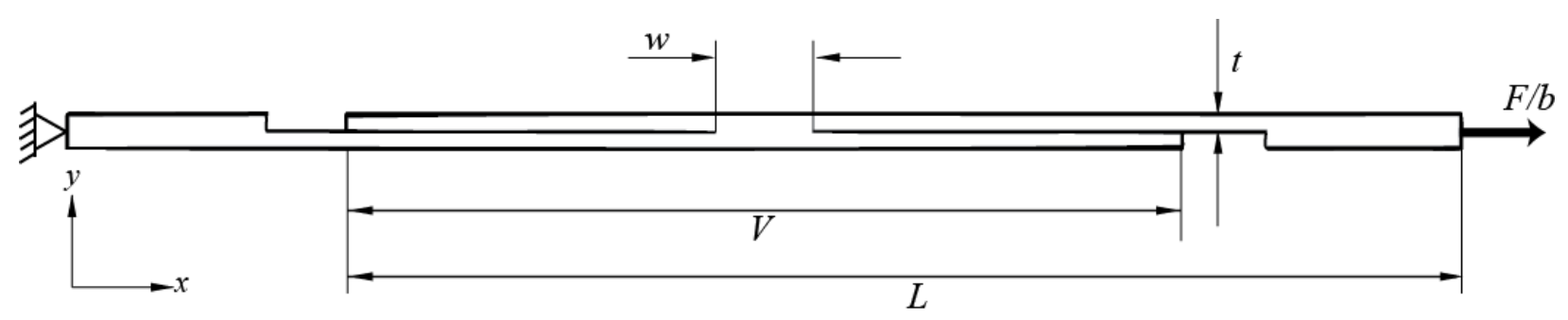

(a)

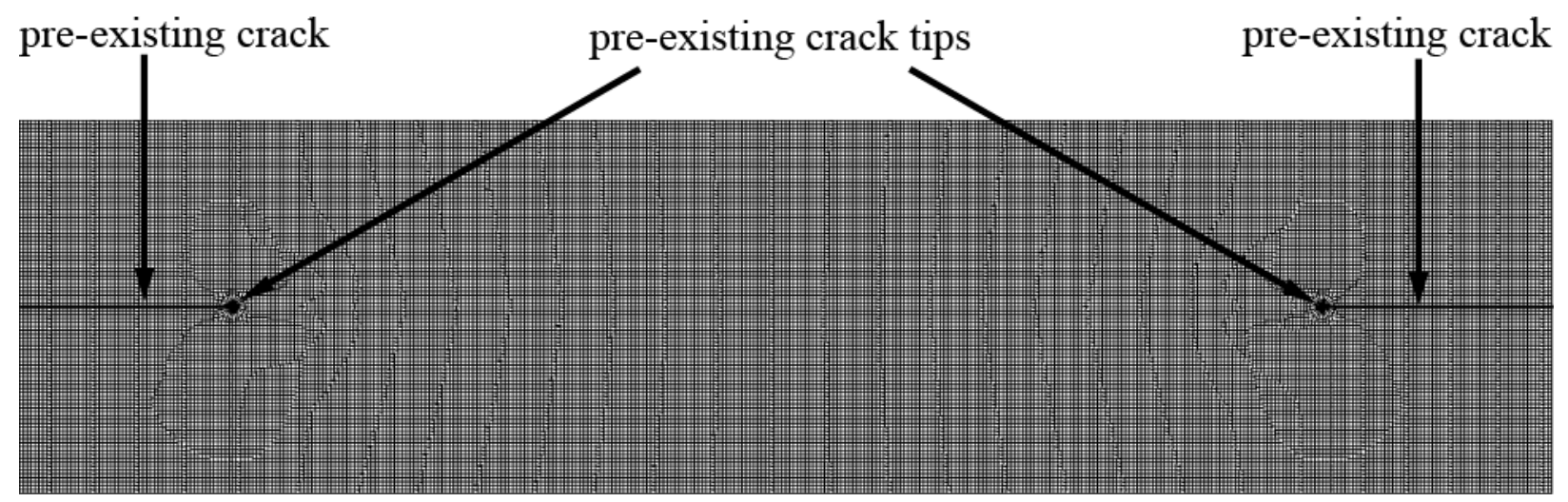

(b)

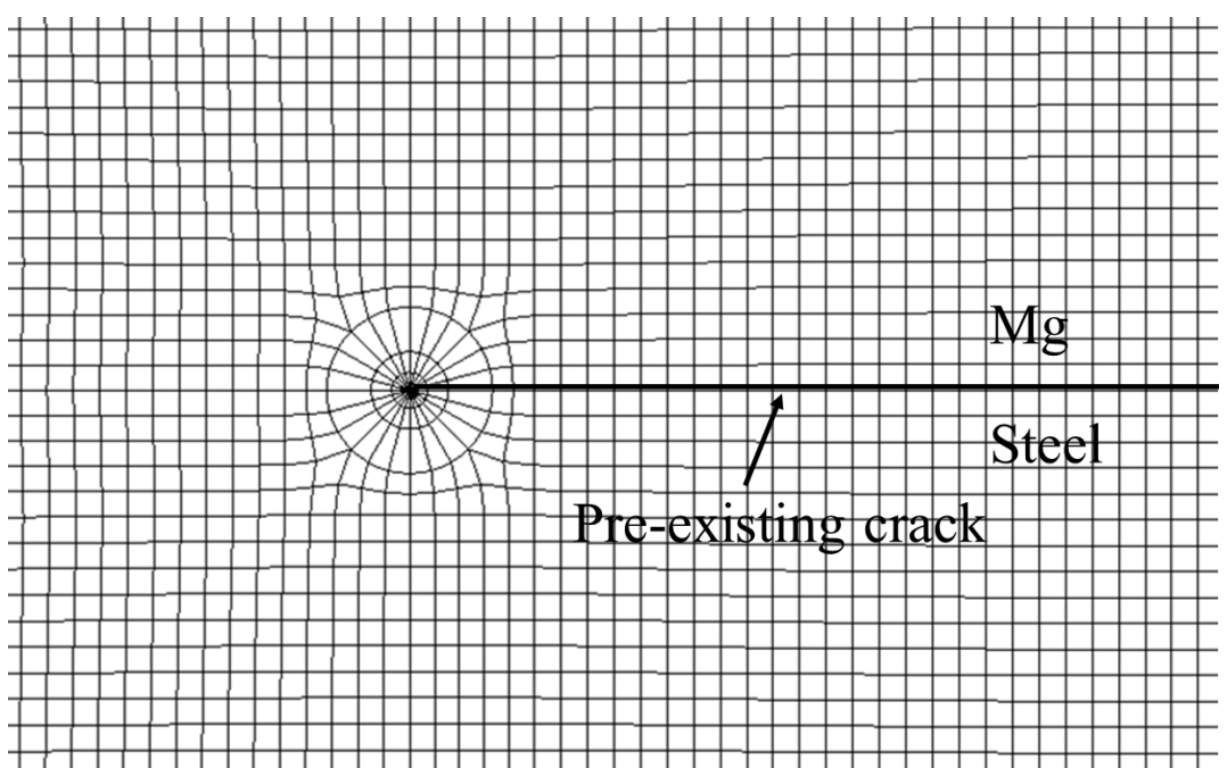

(c)

Figure 5. (a) A schematic of a two-dimensional finite element model of the lap-shear specimen with the boundary and loading conditions. (b) A view of the finite element mesh for the central portion of the finite element mesh and (c) a close-up view of the finite element mesh near the right crack tip for $w / t=5.85$. 
The ratio of the weld width to the thickness is 5.85 for the specimens used in the experiments. The two-dimensional plane strain finite element model is used to find the stress intensity factor solutions which are compared to the solutions in Equations (5) and (6) and those of Erdogan [25]. For future engineering applications, multiple weld widths $w$ are used for the finite element models to understand how the weld width affects the global stress intensity factor solutions. Twelve width to thickness ratios, namely, $w / t=0.0067,0.05,0.125,0.25,0.5,0.64$, $1,2,3,4,5.85$ and 6 were selected in this investigation. Figures 5(b) show the central portion of the finite element mesh and Figure 5(c) shows the mesh surrounding the right main crack tip for $w / t=5.85$. The weld indentation in the upper sheet as shown in Figure 2 is omitted. The effect of the weld indentation was researched by Lee [30] and Franklin [31] for quasi-static loading and fatigue, respectively. The materials in the model are assumed to be homogenous and linear elastic. The top AZ31-H24 sheet is modeled with the Young's modulus $E=45 \mathrm{GPa}$ and the Poisson's ratio $v=0.35$. The bottom HSLA steel sheet is modeled with the Young's modulus $E=206.3 \mathrm{GPa}$ and the Poisson's ratio $v=0.3$. The values of shear modulus $G$ of the magnesium and steel sheets can be calculated by $G=E / 2(1+v)$ as 16.66 and $79.34 \mathrm{GPa}$, respectively. The ratio of the shear modulus of the magnesium sheet to that of the steel sheet is 0.21. This set of the elastic constants is listed in Table 1.

Table 1: Elastic constants of magnesium and steel.

\begin{tabular}{lccc}
\hline & $\begin{array}{c}\text { Elastic } \\
\text { Modulus } \\
E(\mathrm{GPa})\end{array}$ & $\begin{array}{c}\text { Poisson's } \\
\text { ratio }\end{array}$ & $\begin{array}{c}\text { Shear } \\
\text { modulus }\end{array}$ \\
& $45(\mathrm{GPa})$ \\
\hline $\mathrm{Mg}$ & 206.3 & 0.35 & 16.67 \\
Steel & 0.3 & 79.35 \\
\hline
\end{tabular}

The finite element model was meshed using second-order, plane strain, quadrilateral, reduced integration (CPE8R) elements. The $1 / \sqrt{r}$ singularity near the crack tip is modeled using 
second-order quarter point crack-tip elements with collapsed nodes. The calculations were performed using ABAQUS [32].

It should be noted that $K_{1}^{A}$ and $K_{2}^{A}$ of the complex stress intensity factor $\mathbf{K}^{A}\left(=K_{1}^{A}+i K_{2}^{A}\right)$ obtained directly from ABAQUS [32] are defined such that the stresses $\sigma_{y}$ and $\tau_{x y}$ at a small distance $r$ ahead of an interface crack tip are characterized by $\mathbf{K}^{A}$ as

$$
\sigma_{y}+i \tau_{x y}=\frac{K_{1}^{A}+i K_{2}^{A}}{\sqrt{2 \pi r}} r^{i \varepsilon}
$$

The $K_{1}$ and $K_{2}$ solutions as defined in Equation (1) are related to the $K_{1}^{A}$ and $K_{2}^{A}$ solutions as defined in Equation (35) as

$$
\begin{aligned}
& K_{1}=K_{1}^{A} \cos (\varepsilon \ln t)-K_{2}^{A} \sin (\varepsilon \ln t) \\
& K_{2}=K_{1}^{A} \sin (\varepsilon \ln t)+K_{2}^{A} \cos (\varepsilon \ln t)
\end{aligned}
$$

In this investigation, the computational $K_{1}$ and $K_{2}$ solutions for spot welds joining two sheets of dissimilar materials are obtained from Equations (36) and (37) with the $K_{1}^{A}$ and $K_{2}^{A}$ solutions obtained from ABAQUS.

The computational results are compared with the analytical solutions based on the beam bending theory for large ratios of $w / t$ in Equations (5) and (6) and the Erdogan solution [25] for the ratio of $w / t$ approaching to zero in Equations (33) and (34). For comparison of the solutions, the effective stress intensity factor $K_{e}$ is defined as

$$
K_{e}=\sqrt{K_{1}^{2}+K_{2}^{2}}
$$

where $K_{1}$ and $K_{2}$ are obtained from the computational and analytical solutions. Figure 6(a) and 6(b) show a comparison of the normalized effective stress intensity factor $\bar{K}_{e}$ solutions as functions of $w / t$ obtained from the finite element analyses, Equations (5) and (6) based on the 
beam bending theory, and Equations (33) and (34) based on the Erdogan solution in linear and logarithmic scales, respectively. Note that all effective stress intensity factor solutions are normalized by the effective stress intensity factor solutions based on Equations (5) and (6) of the beam bending theory for the right crack tip.

As shown in Figures 6(a) and 6(b), the computational results agree well with the solution of the beam bending theory in Equations (5) and (6) for large ratios of $w / t$. As the ratio $w / t$ decreases, the computational results approach to that of Erdogan. It should be mentioned that although the left and the right cracks have different stress intensity factor solutions, the solutions approach each other as the weld width becomes sufficiently small. A normalized transition weld width can be defined where the effective stress intensity factor $\bar{K}_{e}$ of the beam bending theory is equal to that of Erdogan. For the right crack, the normalized transition weld width occurs at $w / t=0.209$. For the left crack, the normalized transition weld width occurs at $w / t=1.216$. The transition does not occur at the same $w / t$ ratio for the two cracks. The normalized transition weld width is smaller for the right crack with the higher effective stress intensity factor. It should be noted that the normalized transition weld width for dissimilar welds in lapshear specimens depends on the particular combination of materials. Sripichai et al. [16] found the normalized transition weld width is 0.364 for similar welds in lap-shear specimens of equal thickness. Generally speaking, the transition is expected to be at a small normalized width ratio. Table 2 lists the normalized computational $\bar{K}_{1}, \bar{K}_{2}$ and $\bar{K}_{e}$ solutions as functions of $w / t$ for future engineering applications. The $\bar{K}_{1}$ and $\bar{K}_{2}$ solutions are normalized by the analytical $\left|K_{2}\right|$ solution for the right crack in Equation (6). The $\bar{K}_{e}$ solutions are normalized by the effective stress intensity factor solutions based on Equations (5) and (6) for the right crack. 


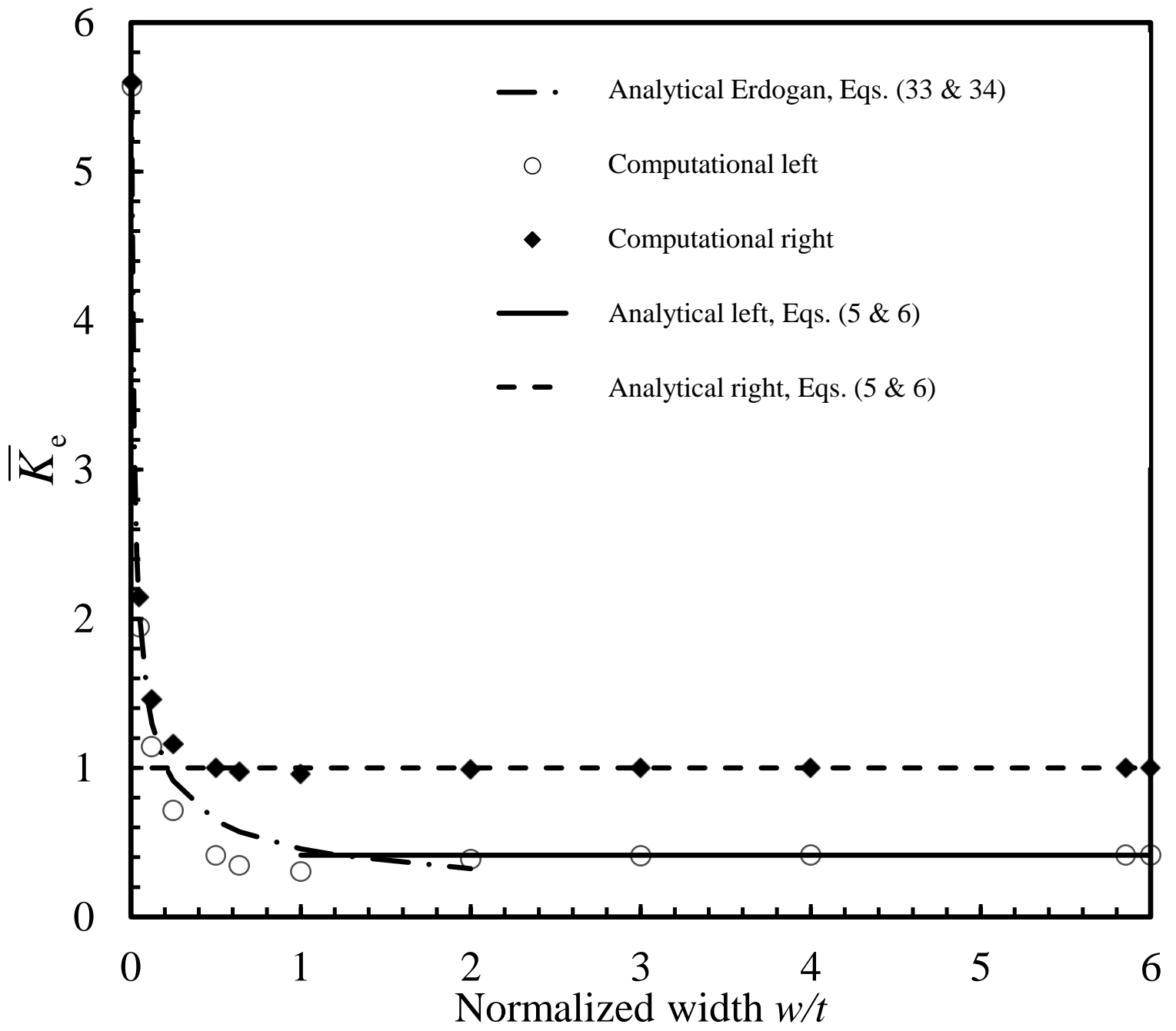

(a) 


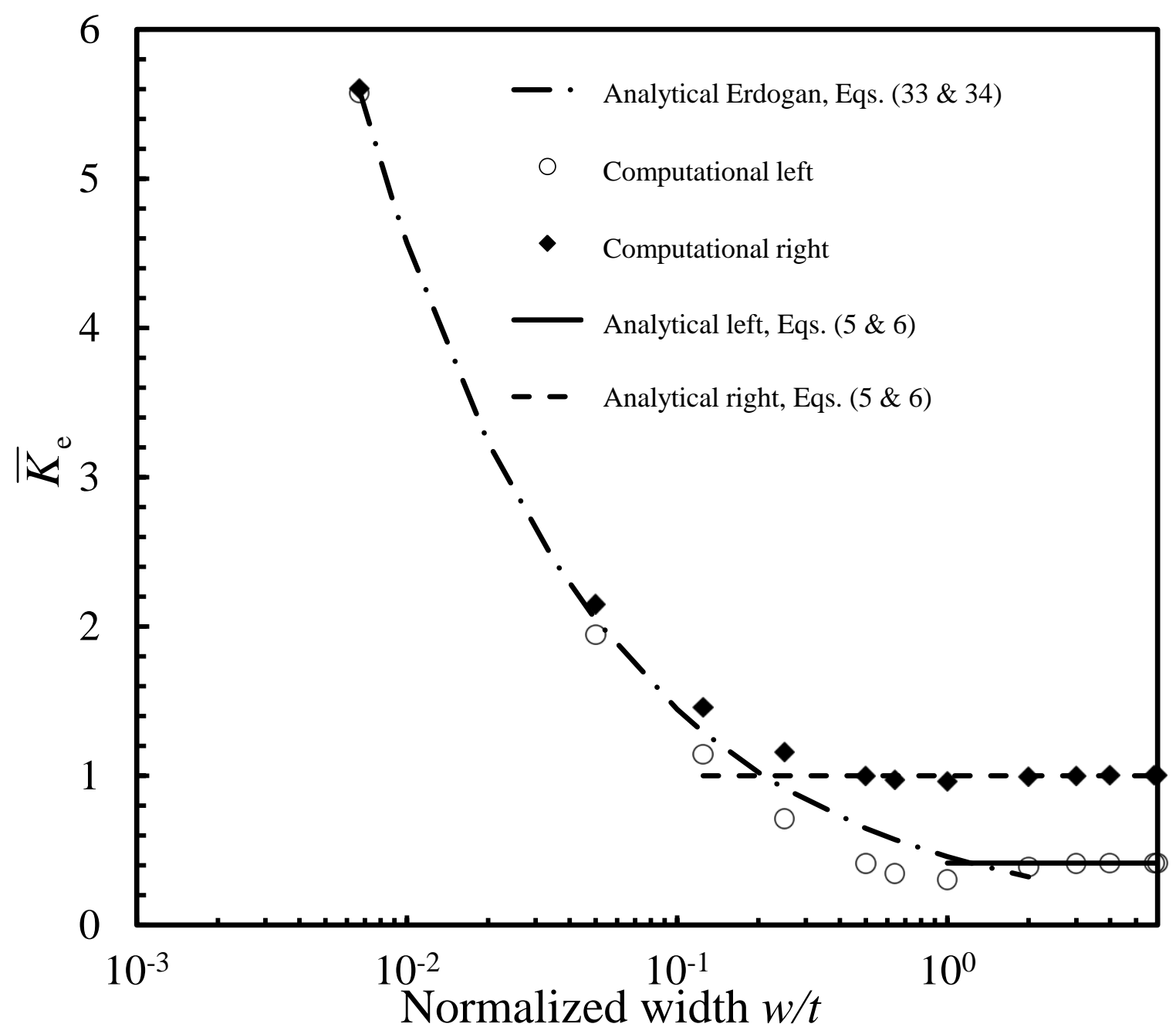

(b)

Figure 6. The normalized effective stress intensity factor solutions as functions of $w / t$ plotted in (a) linear and (b) logarithmic scales.

Table 2a: The normalized computational global stress intensity factor $\bar{K}_{1}, \bar{K}_{2}$ and $\bar{K}_{\mathrm{e}}$ solutions for the left crack for various normalized weld widths.

\begin{tabular}{ccccccc}
\hline$w / t$ & 0.0067 & 0.05 & 0.125 & 0.25 & 0.5 & 0.64 \\
$\bar{K}_{1, \text { left }}$ & -2.633 & -0.726 & -0.370 & -0.187 & -0.025 & 0.037 \\
$\left|\bar{K}_{2}\right|_{\text {left }}$ & 6.378 & 2.296 & 1.365 & 0.864 & 0.510 & 0.425 \\
$\bar{K}_{\mathrm{e}, \text { left }}$ & 5.572 & 1.944 & 1.142 & 0.714 & 0.412 & 0.344 \\
\hline
\end{tabular}


Table 2 a continued

\begin{tabular}{ccccccc}
\hline$w / t$ & 1 & 2 & 3 & 4 & 5.85 & 6 \\
$\bar{K}_{1, \text { left }}$ & 0.161 & 0.328 & 0.355 & 0.358 & 0.358 & 0.358 \\
$\left|\bar{K}_{2}\right|_{\text {left }}$ & 0.339 & 0.351 & 0.366 & 0.368 & 0.368 & 0.368 \\
$\bar{K}_{\text {e, left }}$ & 0.303 & 0.388 & 0.412 & 0.414 & 0.415 & 0.415 \\
\hline
\end{tabular}

Table 2b: The normalized computational global stress intensity factor $\bar{K}_{1}, \bar{K}_{2}$ and $\bar{K}_{\mathrm{e}}$ solutions for the right crack for various normalized weld widths.

\begin{tabular}{ccccccc}
\hline$w / t$ & 0.0067 & 0.05 & 0.125 & 0.25 & 0.5 & 0.64 \\
$\bar{K}_{1, \text { right }}$ & 2.643 & 0.787 & 0.468 & 0.348 & 0.331 & 0.357 \\
$\left|\bar{K}_{2}\right|_{\text {right }}$ & 6.412 & 2.540 & 1.746 & 1.390 & 1.193 & 1.152 \\
$\bar{K}_{\text {e,right }}$ & 5.600 & 2.147 & 1.460 & 1.157 & 1.000 & 0.974 \\
\hline
\end{tabular}

Table $2 \mathrm{~b}$ continued

\begin{tabular}{ccccccc}
\hline$w / t$ & 1 & 2 & 3 & 4 & 5.85 & 6 \\
$\bar{K}_{1, \text { right }}$ & 0.454 & 0.666 & 0.719 & 0.728 & 0.729 & 0.729 \\
$\left|\bar{K}_{2}\right|_{\text {right }}$ & 1.099 & 1.029 & 1.006 & 1.003 & 1.002 & 1.002 \\
$\bar{K}_{\text {e,right }}$ & 0.960 & 0.990 & 0.999 & 1.001 & 1.001 & 1.001 \\
\hline
\end{tabular}

The near-tip stresses for main cracks are also examined. Figure 7 shows a magnified view of the finite element model near the right crack tip for the lap-shear specimen with $w / t=5.85$. The loading and boundary conditions are the same as indicated in Figure 5 (a). The crack-tip stresses are obtained as functions of the radial distance to the tip, $r$, directly ahead of and above the crack tip. 


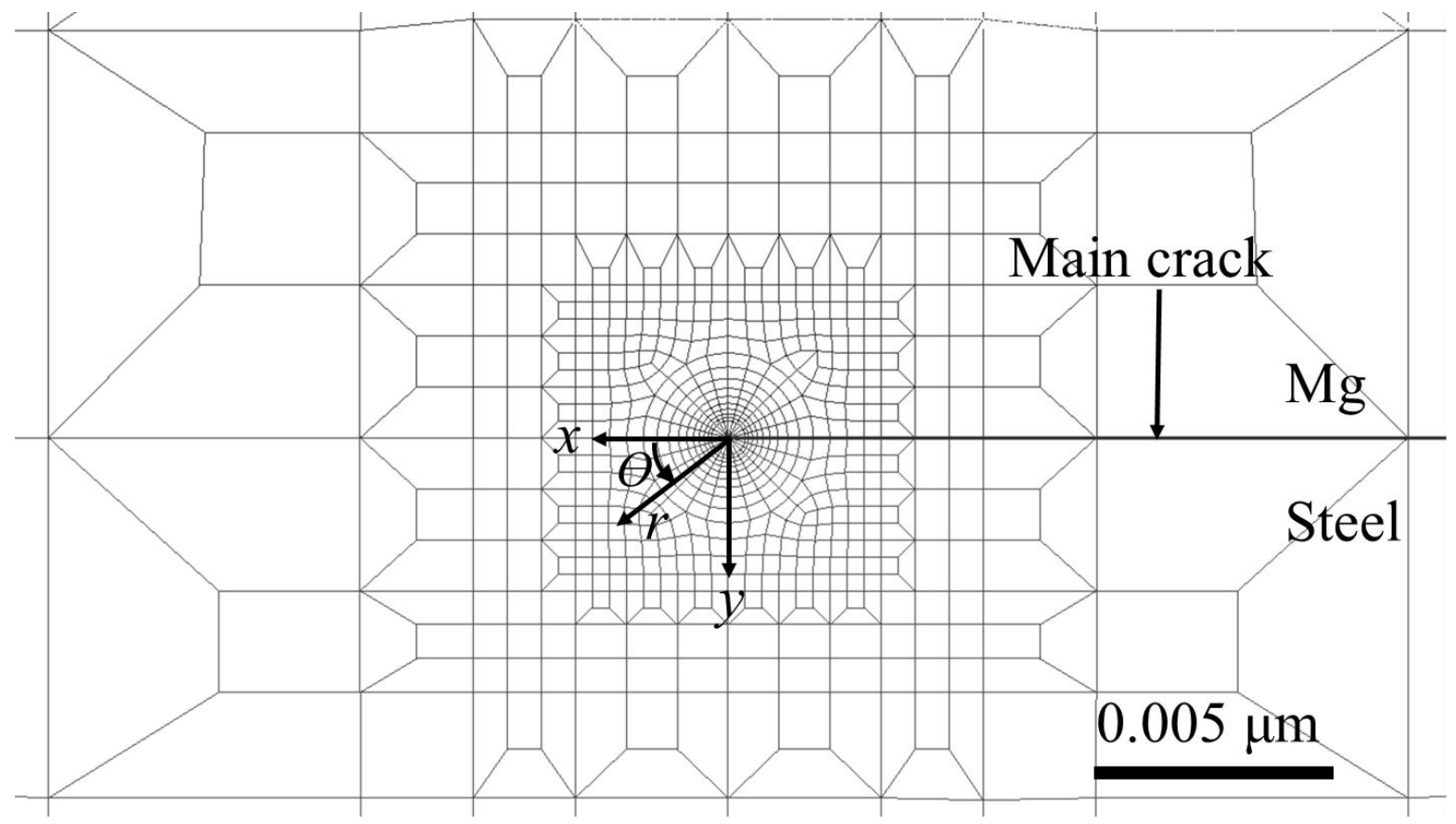

Figure 7. The finite element mesh near the right main crack indicating the directions ahead of the crack tip $\theta=0^{\circ}$, and above the crack tip $\theta=-90^{\circ}$, as pointing horizontally and vertically from the main crack tip, respectively.

A second finite element analysis is also carried out on the same computational model with a set of modified elastic constants of $E=48.6 \mathrm{GPa}, v=0.458$ and $G=16.7 \mathrm{GPa}$ for the magnesium sheet and $E=206.3 \mathrm{GPa}, v=0.3$ and $G=79.3 \mathrm{GPa}$ for the steel sheet. This set of elastic constants is listed in Table 3.

Table 3: Modified elastic constants for magnesium.

\begin{tabular}{lccc}
\hline & $\begin{array}{c}\text { Elastic } \\
\text { Modulus } \\
E(\mathrm{GPa})\end{array}$ & $\begin{array}{c}\text { Poisson's } \\
\text { ratio }\end{array}$ & $\begin{array}{c}\text { Shear } \\
\text { modulus }\end{array}$ \\
& $v$ & $G(\mathrm{GPa})$ \\
\hline Modified $\mathrm{Mg}$ & 48.6 & 0.458 & 16.67 \\
Steel & 206.3 & 0.3 & 79.35 \\
\hline
\end{tabular}

This set of elastic constants gives the same ratio of the shear moduli as the actual elastic constants. The Young's modulus and Poisson's ratio of the magnesium sheet are modified to give $\beta=0$ and consequently $\varepsilon=0$. Here, the steel elastic constants are kept the same as those 
for the actual material. It would be preferred to keep the actual elastic constants for the magnesium sheet to give $\beta=0$. However, this would result in a negative Poisson's ratio for the steel. The crack-tip stresses ahead of and above the crack tip are compared for the solutions based on the actual and modified elastic constants in Table 1 and Table 3 respectively. The computational results based on the modified elastic constants are used as the reference to examine the asymptotic behavior of the crack-tip stresses for $\beta \neq 0$ and $\varepsilon \neq 0$.

Figures 8(a) and 8(b) show the normalized opening and shear stresses $\sigma_{22} / \bar{\sigma}$ and $\sigma_{12} / \bar{\sigma}$ as functions of the normalized radius $r / t$ directly ahead of the crack tip. Here, the stresses are normalized by $\bar{\sigma}(=F /(b t))$ which represents the normalized applied stress. As shown in the figures, the normalized opening and shear stresses $\sigma_{22} / \bar{\sigma}$ and $\sigma_{12} / \bar{\sigma}$ directly ahead of the crack tip for the two sets of material elastic constants are different when the normalized radius decreases to small values due to the oscillation of the stresses near the crack tip for the results based on the actual elastic constants for the magnesium and steel. For the results based on the modified elastic constants ( $\beta=0$ or $\varepsilon=0$ ), the crack tip stresses follow the usual $1 / \sqrt{r}$ singularity. Figures 8(c) and 8(d) show the normalized stresses $\sigma_{11} / \bar{\sigma}$ and $\sigma_{12} / \bar{\sigma}$ as functions of the normalized radius $r / t$ above the crack tip for the two sets of elastic constants. As shown in the figures, the dominant normalized opening stress $\sigma_{11} / \bar{\sigma}$ appears to follow the $1 / \sqrt{r}$ singularity quite consistently for both sets of elastic constants while the normalized shear stress $\sigma_{12} / \bar{\sigma}$ remains slightly influenced by the oscillation of the actual material with $\beta \neq 0$ or $\varepsilon \neq 0$. The crack-tip stress shown in Figures 8(a)-(b) indicate that the oscillations of the crack-tip stresses appear to be stronger directly ahead of the tip. 


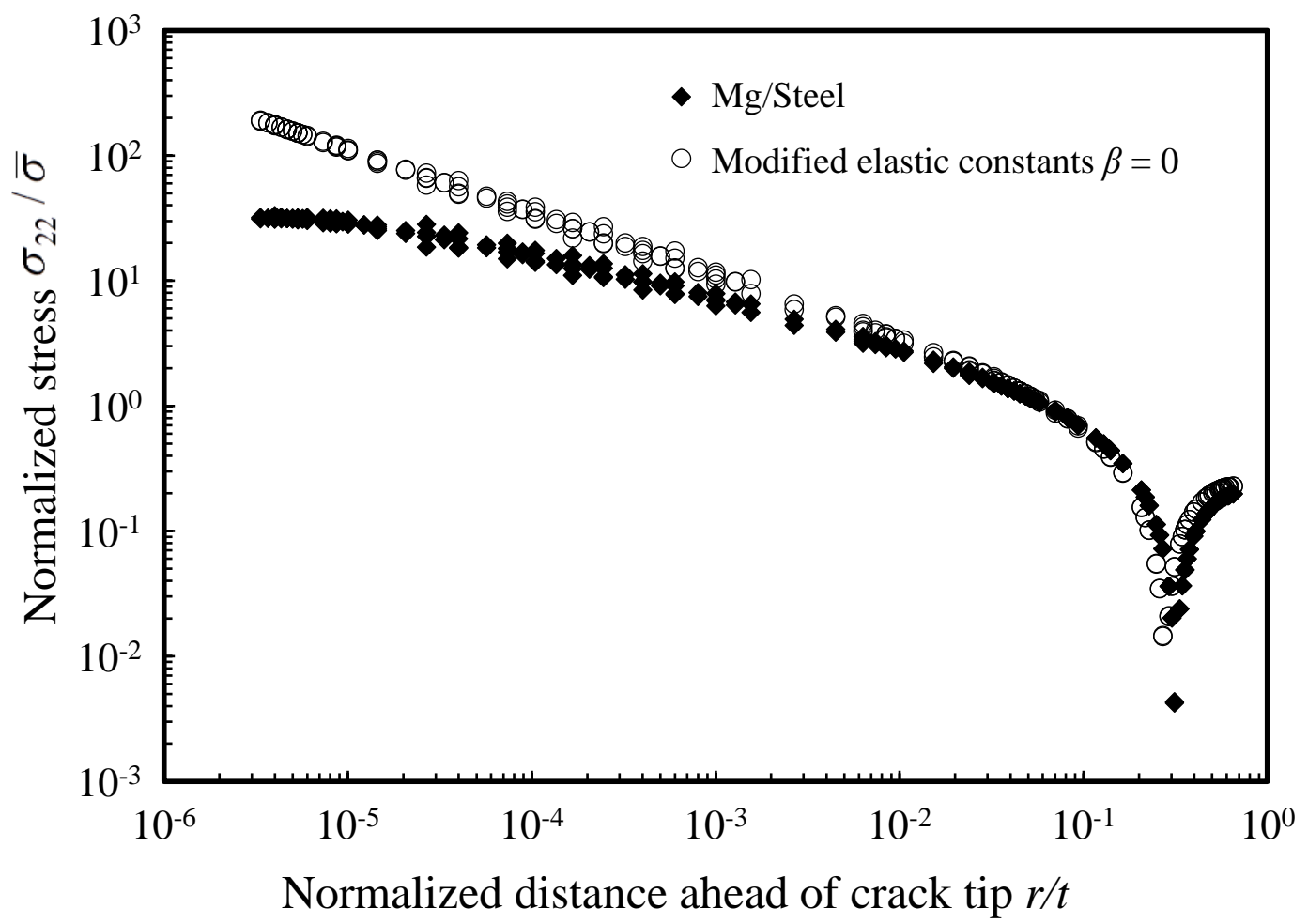

(a)

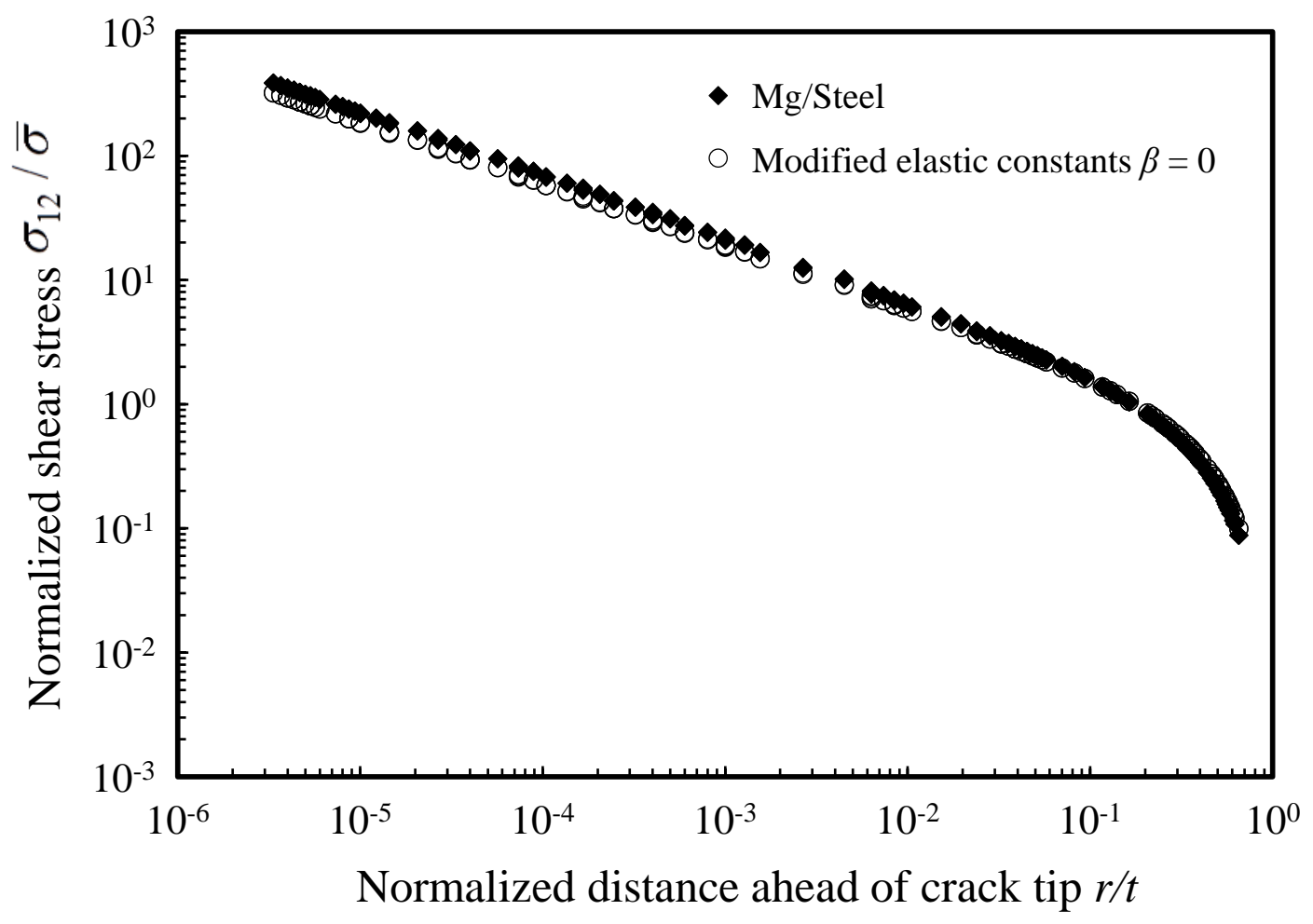

(b) 


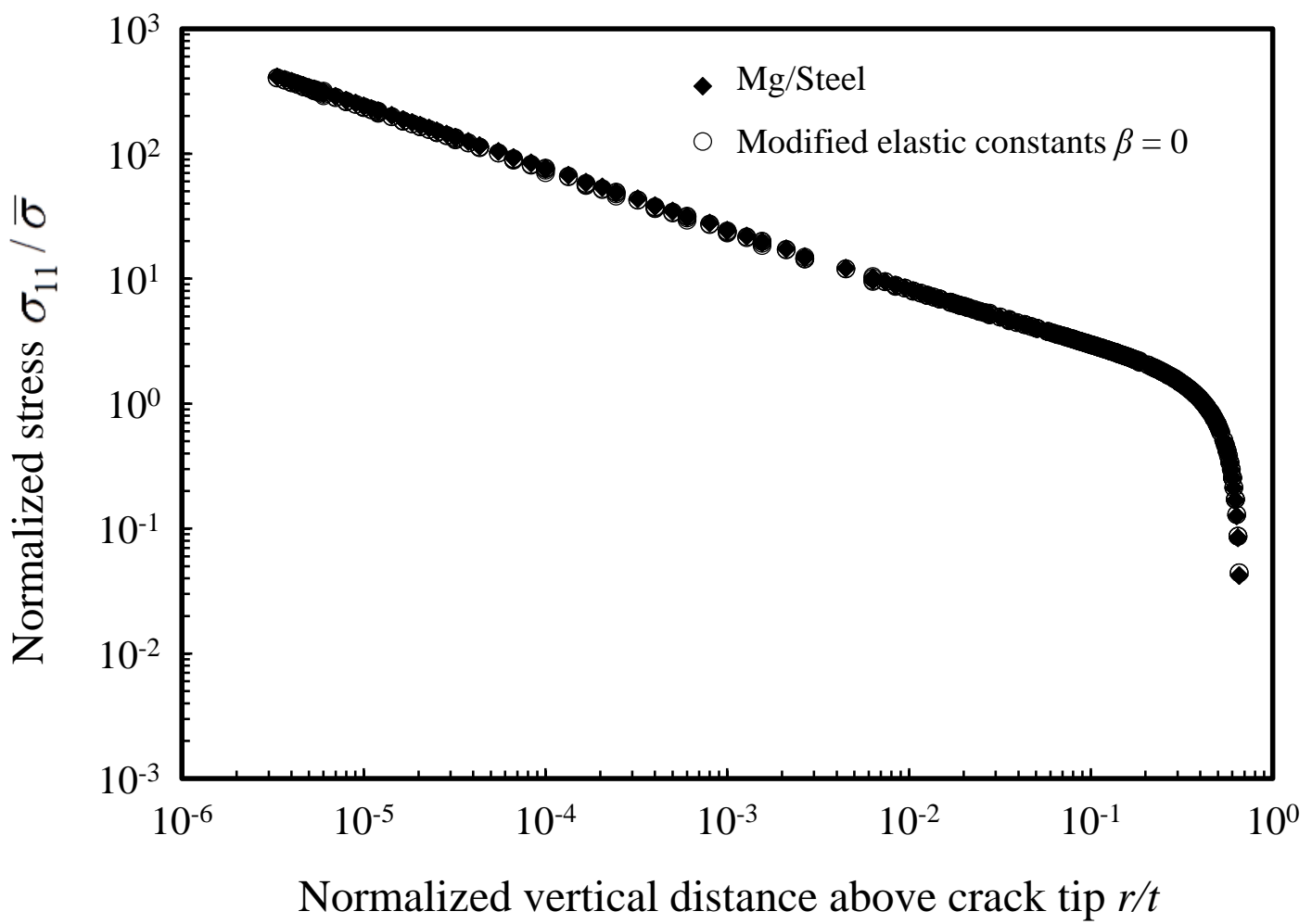

(c)

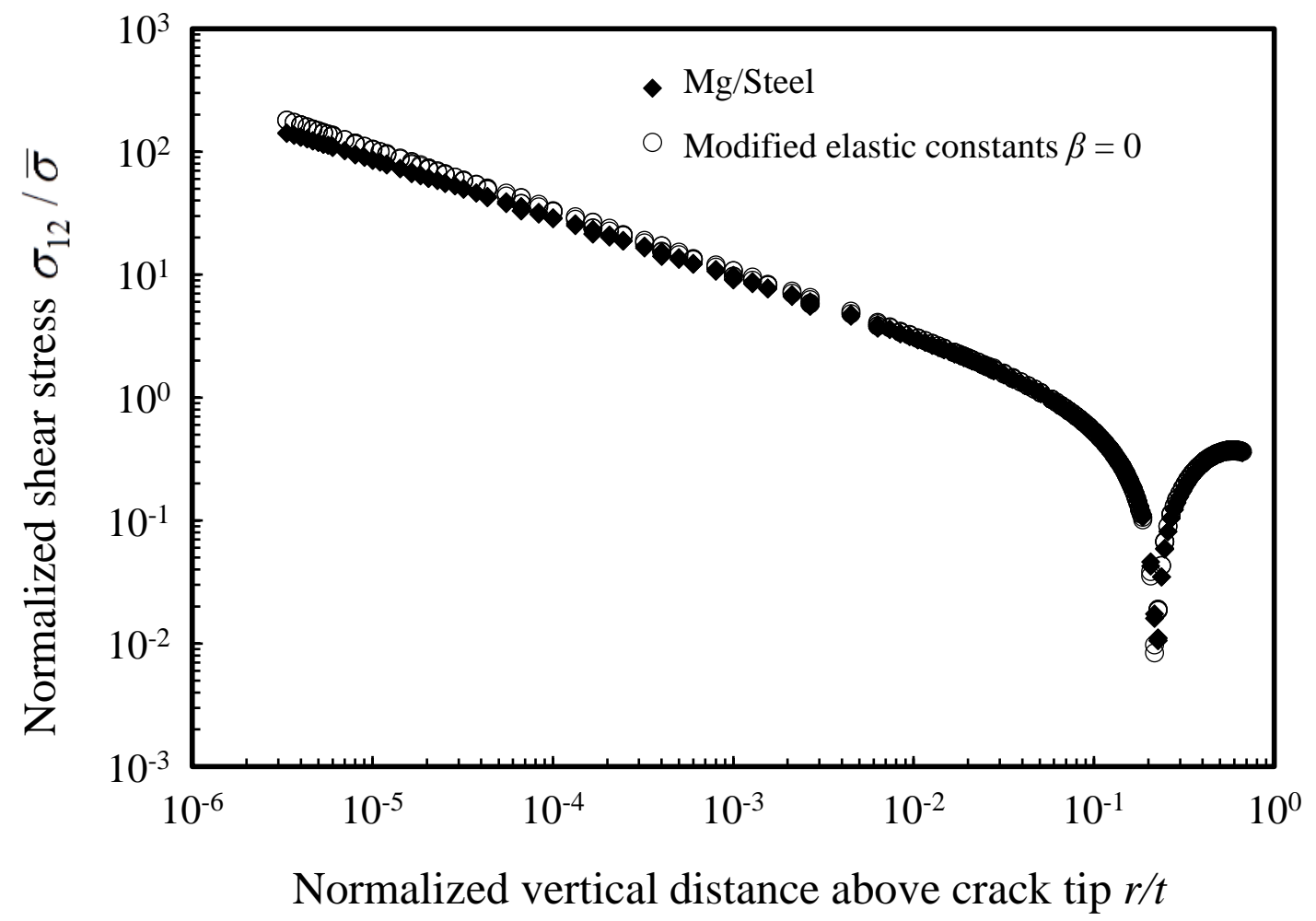

(d) 
Figure 8. A comparison of (a) the normal stress in the $y$ direction ahead of the crack tip, (b) the shear stress ahead of the crack tip, (c) the normal stress in the $x$ direction vertically above the crack tip, and (d) the shear stress vertically above the crack tip from the finite element analyses based on the actual elastic constants and the modified elastic constants.

The crack-tip opening stresses above the crack tip in the vertical direction does not appear to be influenced by the oscillation as the crack-tip stresses directly ahead of the crack tip. Note that from the experimental observations, kinked cracks grew in the vertical direction above the crack tip. This suggests that the local stress intensity factor solutions for the modified elastic constants with $\beta=0$ or $\varepsilon=0$ may be used for the kinked crack with the vanishing kink length.

\section{Analytical local stress intensity factor solutions for kinked cracks}

A schematic of a main crack and a kinked crack with the kink length $a$ and the kink angle $\varphi$ is shown in Figure 9. The local stress intensity factor solutions for the kinked crack are $k_{\mathrm{I}}$ and $k_{\mathrm{II}}$. The global stress intensity factor solutions for the main crack are $K_{1}$ and $K_{2}$. The arrows in the figure show the positive direction for the local stress intensity factors $k_{\mathrm{I}}$ and $k_{\mathrm{II}}$.

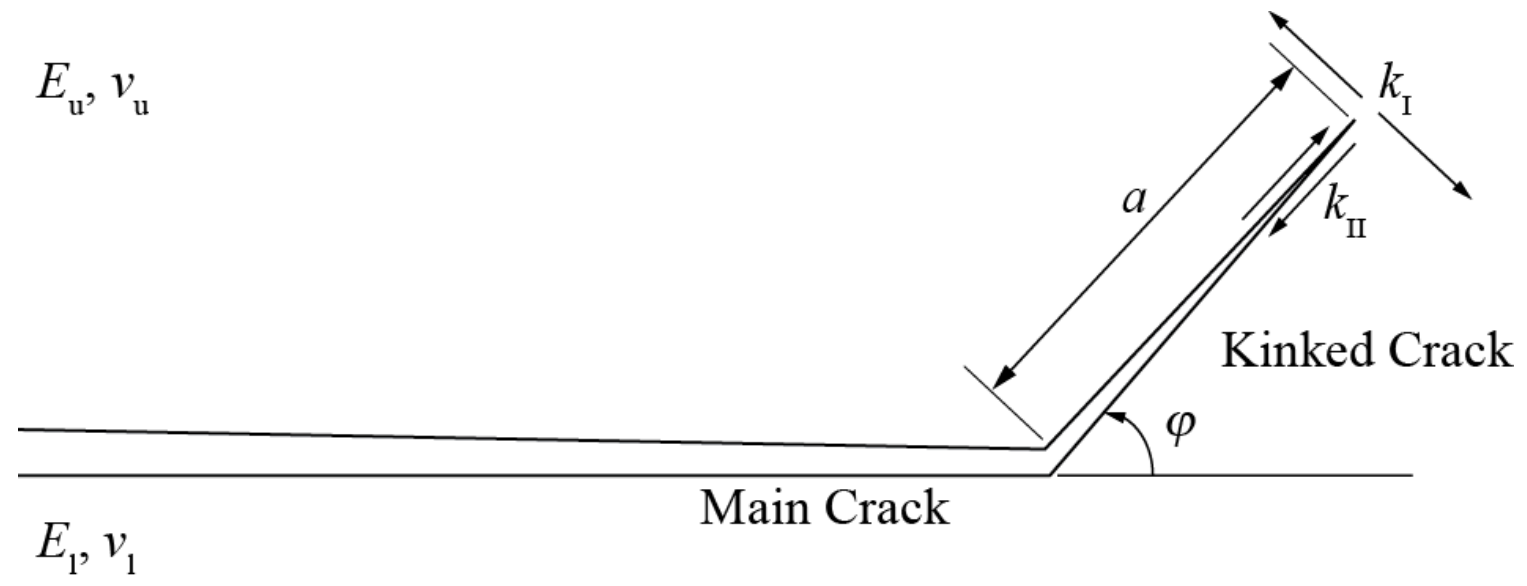

Figure 9. A schematic of a main crack and a kinked crack with the kink length $a$ and the kink angle $\varphi$. 
For kink lengths approaching to 0 in dissimilar material, the $k_{\mathrm{I}}$ and $k_{\mathrm{II}}$ solutions can be expressed as functions of the Dundurs' parameters $\alpha$ and $\beta$, the kink angle $\varphi$, and the global stress intensity factors $K_{1}$ and $K_{2}$ for the main crack. The local stress intensity factors $k_{\mathrm{I}}$ and $k_{\text {II }}$ are expressed in the complex form (He and Hutchinson [24]) as

$$
k_{I}+i k_{I I}=\left(c_{R}+i c_{I}\right)\left(K_{1}+i K_{2}\right) a^{i \varepsilon}+\left(d_{R}-i d_{I}\right)\left(K_{1}-i K_{2}\right) a^{-i \varepsilon}
$$

where $c_{R}, c_{I}, d_{R}$ and $d_{I}$ are the real and imaginary part of the complex function $c$ and $d$.

Both $c$ and $d$ are complex functions of $\alpha, \beta$ and $\varphi$. Equation (39) indicates that $k_{\mathrm{I}}$ and $k_{\mathrm{II}}$ depend on the crack length $a$ and bimaterial constant $\varepsilon$. The global stress intensity factors $K_{1}$ and $K_{2}$ are defined in the form as in Equation (35).

The functions $c_{R}, c_{I}, d_{R}$ and $d_{I}$ were tabulated in He and Hutchinson [33]. The values for $c$ and $d$ were interpolated from that report and are listed in Table 4.

Table 4: Values of $c_{R}, c_{I}, d_{R}$ and $d_{I}$ for $\mathrm{Mg} / \mathrm{Steel}$ material and material with modified elastic constants interpolated from [33].

\begin{tabular}{lcccc}
\hline & $c_{R}$ & $c_{I}$ & $d_{R}$ & $d_{I}$ \\
\hline $\mathrm{Mg} /$ Steel Material & 0.100 & -0.697 & 0.198 & -0.378 \\
Modified Elastic Constants for $\beta=0$ & 0.109 & -0.709 & 0.233 & -0.398 \\
\hline
\end{tabular}

As indicated in Equation (39), when the crack length approaches zero, the values for the local stress intensity factor solutions depend on the value of $a$ when the bimaterial constant $\varepsilon$ is nonzero. The dependence of the solutions on the crack length $a$ presents a challenge to develop a fatigue life estimation method based on a fatigue crack growth model, even though the oscillation is in a region which is too small to be of concern. This challenge prevents the evaluation of the limit at the crack length of $a=0$ in Equation (39). 
However, for $\varepsilon=0$, Equation (39) simplifies to

$$
\begin{aligned}
& \left(k_{I}\right)_{0}=\left(c_{R}+d_{R}\right) K_{1}-\left(c_{I}+d_{I}\right) K_{2} \\
& \left(k_{I I}\right)_{0}=\left(c_{I}-d_{I}\right) K_{1}+\left(c_{R}-d_{R}\right) K_{2}
\end{aligned}
$$

where $\left(k_{I}\right)_{0}$ and $\left(k_{I I}\right)_{0}$ represent the mode I and II stress intensity factor solution at the kink length $a$ equal to zero. For cracks between similar materials, Equations (40) and (41) are reduced to those of Cotterell and Rice [34]. It should be noted that for $\varepsilon=0$, the crack-tip stresses recover the $1 / \sqrt{r}$ singularity.

The local stress intensity factor solutions for finite crack length kinked cracks in lap-shear specimens can be expressed as functions of the normalized kink length $a / t$ as in [16] as

$$
\begin{aligned}
& k_{\mathrm{I}}(a / t)=f_{\mathrm{I}}(a / t, w / t) \cdot \frac{F}{b \sqrt{t}} \\
& k_{\mathrm{II}}(a / t)=f_{\mathrm{II}}(a / t, w / t) \cdot \frac{F}{b \sqrt{t}} \\
& k_{\mathrm{e}}(a / t)=f_{\mathrm{e}}(a / t, w / t) \cdot \frac{F}{b \sqrt{t}}
\end{aligned}
$$

where $f_{\mathrm{I}}, f_{\mathrm{II}}$ and $f_{e}$ are geometric functions which depend on the crack length $a$, the weld width $w$ and the sheet thickness $t$ for the given material combination. The local stress intensity factor solutions for idealized dissimilar welds in lap-shear specimens were obtained computationally for $w / t=5.85$ to match that of the lap-shear specimen used in the experiments and for $w / t=1$ and 0.5 for future engineering applications.

\section{Computational local stress intensity factor solutions for finite kinked cracks}


Sripichai et al. [16] obtained the local stress intensity factor solutions for kinked cracks of different kink lengths by two-dimensional plane strain finite element analyses for welds of similar material with $w / t=0.5,1$ and 2 . In this investigation, two-dimensional plane strain finite element analyses were performed for dissimilar welds in the lap-shear specimens to obtain the local stress intensity factor solutions for kinked cracks as functions of kink lengths for $w / t=0.5,1$ and 5.85. Fourteen normalized kink lengths, $a / t=0.00093,0.002,0.005,0.01$, $0.025,0.05,0.075,0.1,0.2,0.3,0.4,0.5,0.6$ and 0.7 were modeled. First, the local stress intensity factor solutions are obtained computationally for $w / t=5.85$ based on the actual elastic constants. These computational results are compared with the solutions for the vanishing crack length in Equation (39) for the actual elastic constants and in Equations (40) and (41) for the modified elastic constants. The stress intensity factor solutions for the vanishing crack length in Equation (39) are based on the computational $K_{1}$ and $K_{2}$ solutions for the main crack using the actual elastic constants. The solutions in Equations (40) and (41) are based on the computational $K_{1}$ and $K_{2}$ solutions for the main crack using the modified elastic constants. Then, the local stress intensity factor solutions for kinked cracks of normalized widths $w / t=0.5,1$ and 5.85 will be investigated.

The schematic for a two-dimensional finite element model of a lap-shear specimen with a kinked crack is show in Figure 10(a) with the boundary and loading conditions similar to those for the main crack investigation in Figure 5(a). The lap-shear specimen is modeled for the weld widths of $w=0.75,1.5$ and $8.78 \mathrm{~mm}$ that correspond to $w / t=0.5,1$ and 5.85 , respectively. For $w / t=5.85$, the finite element analyses were carried out based on the actual elastic constants with the additional normalized kink length $a / t=6.67 \times 10^{-6}$ for comparison as the crack length approaches zero. The width $b$ of the reduced section in the middle of the specimen is $8 \mathrm{~mm}$. 
The length $L$ of the upper and lower sheets and the overlap distance $V$ remained unchanged as $100 \mathrm{~mm}$ and $75 \mathrm{~mm}$, respectively. Both upper and lower sheets are modeled with an idealized thickness of $1.5 \mathrm{~mm}$. In Figure 10(a), a kinked crack is schematically shown to grow from the right crack tip into the upper magnesium sheet. Based on the micrograph in Figure 2, the value of the kink angle is selected to be $90^{\circ}$ for this investigation. It should be noted that according to the sign convention in Figure 9, the kinked angle $\varphi$ should be $-90^{\circ}$. Figure 10(b) shows the finite element mesh near the right crack tip for $w / t=5.85$ and $a / t=0.1$. Figures $10(\mathrm{c})$ and 10(d) show the mesh near the right main crack and a closer view of the finite element mesh near the kinked crack tip, respectively.

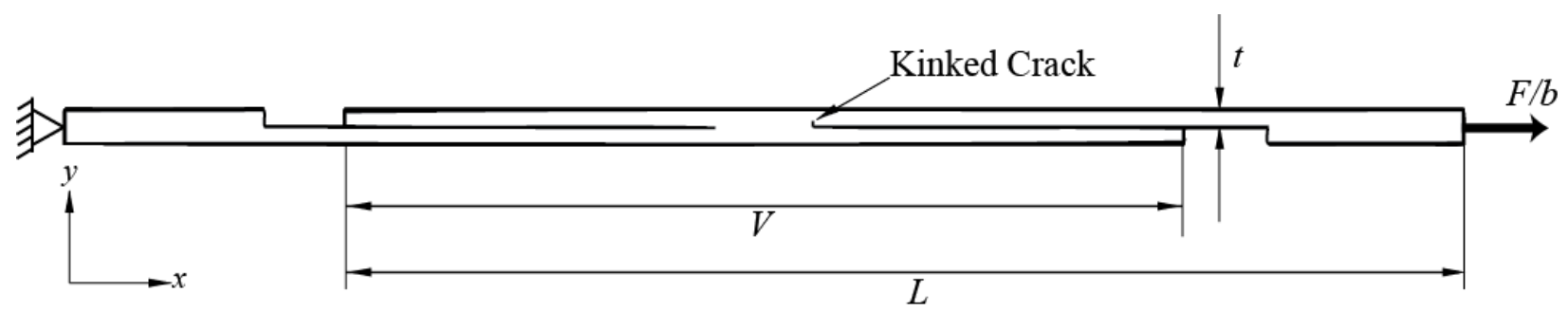




\section{Pre-existing crack}

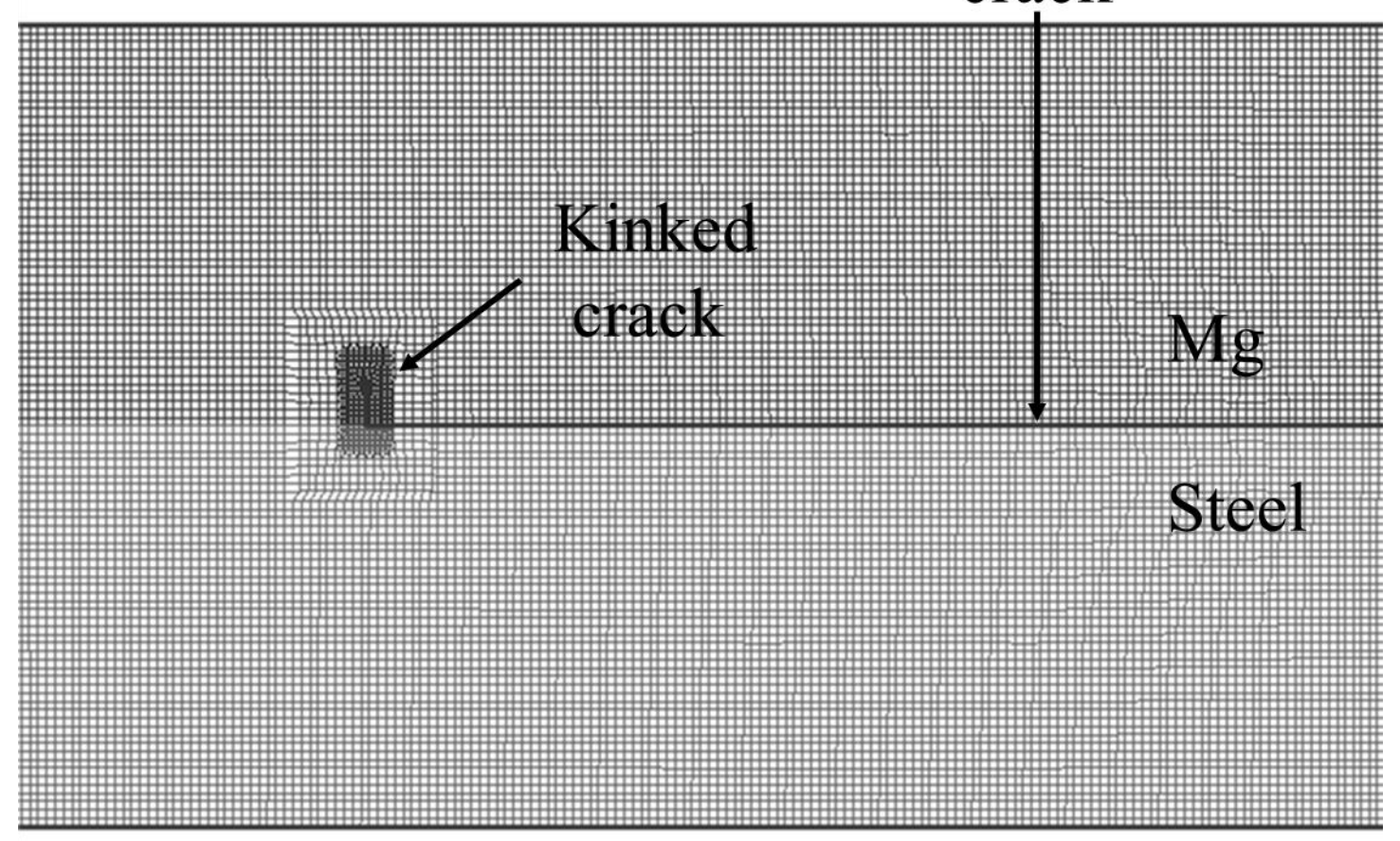

(b)

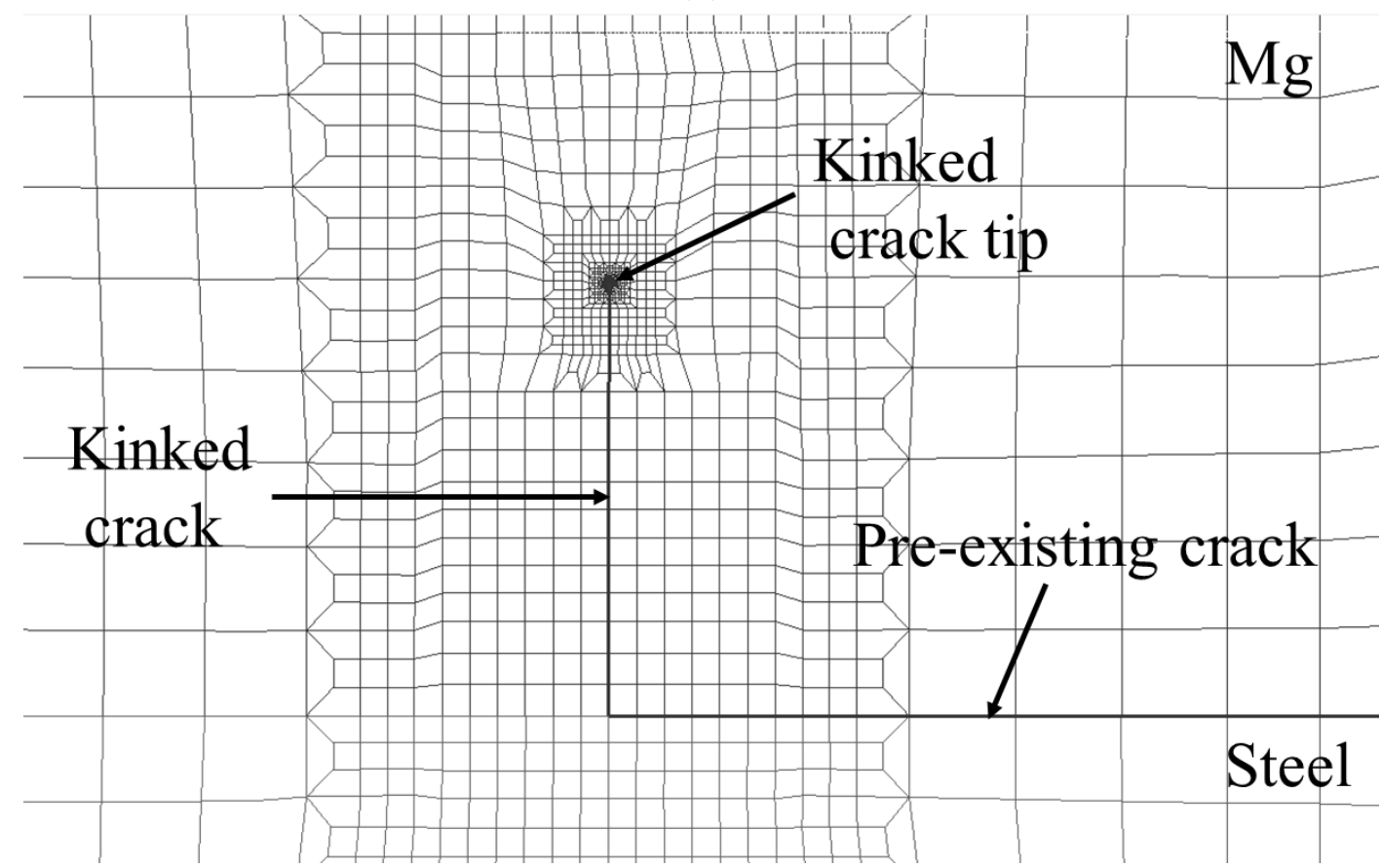

(c) 


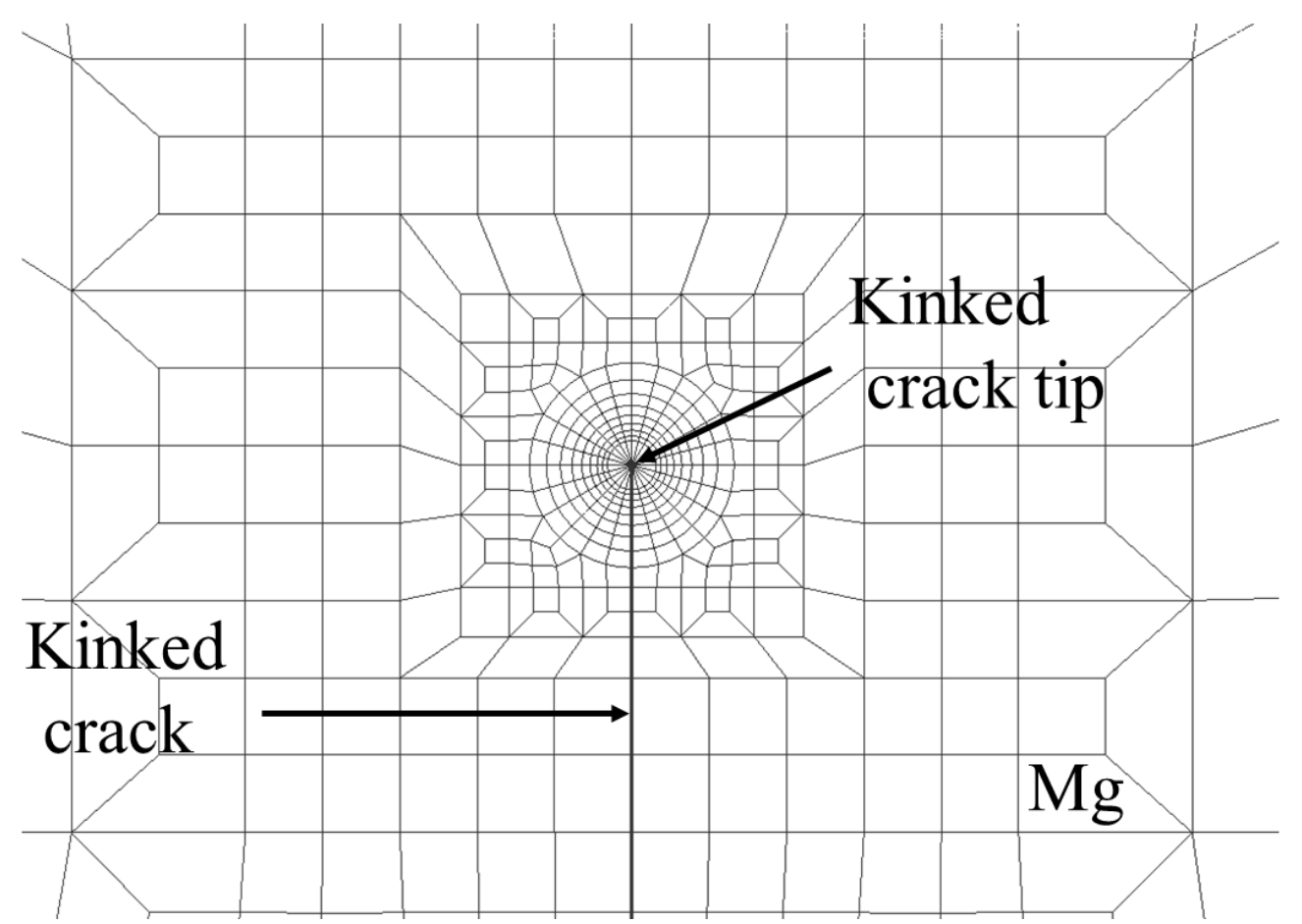

(d)

Figure 10. (a) A schematic of a two-dimensional finite elemental model of a lap-shear specimen with a kinked crack with the boundary and loading conditions. (b) The finite element mesh showing the pre-existing cracks and the kinked crack on the right hand side for $w / t=5.85$ and $a / t=0.1$, (c) the finite element mesh near the right main crack tip, and (d) a close-up view of the finite element mesh near the kinked crack tip.

Figures 11(a) and 11(b) show the computational results and analytical solutions for $f_{\mathrm{I}}$ as functions of the normalized kink length $a / t$ for $w / t=5.85$ based on the actual elastic constants in linear and logarithmic scales, respectively. Also shown in the figures are the analytic solutions for small kink lengths in Equation (39) based on the actual elastic constants and the analytical solutions for the vanishing kink length in Equation (40) based on the modified elastic constants. The kinked crack solution in Equation (39) based on the actual elastic constants decreases as the kinked crack length decreases and does not appear to reach a limit. The kinked crack solution in Equation (40) based on the modified elastic constants is constant for all crack 
lengths. This vanishing kinked crack solution correlates well with the computational $f_{\mathrm{I}}$ solutions based on both the actual and modified elastic constants.

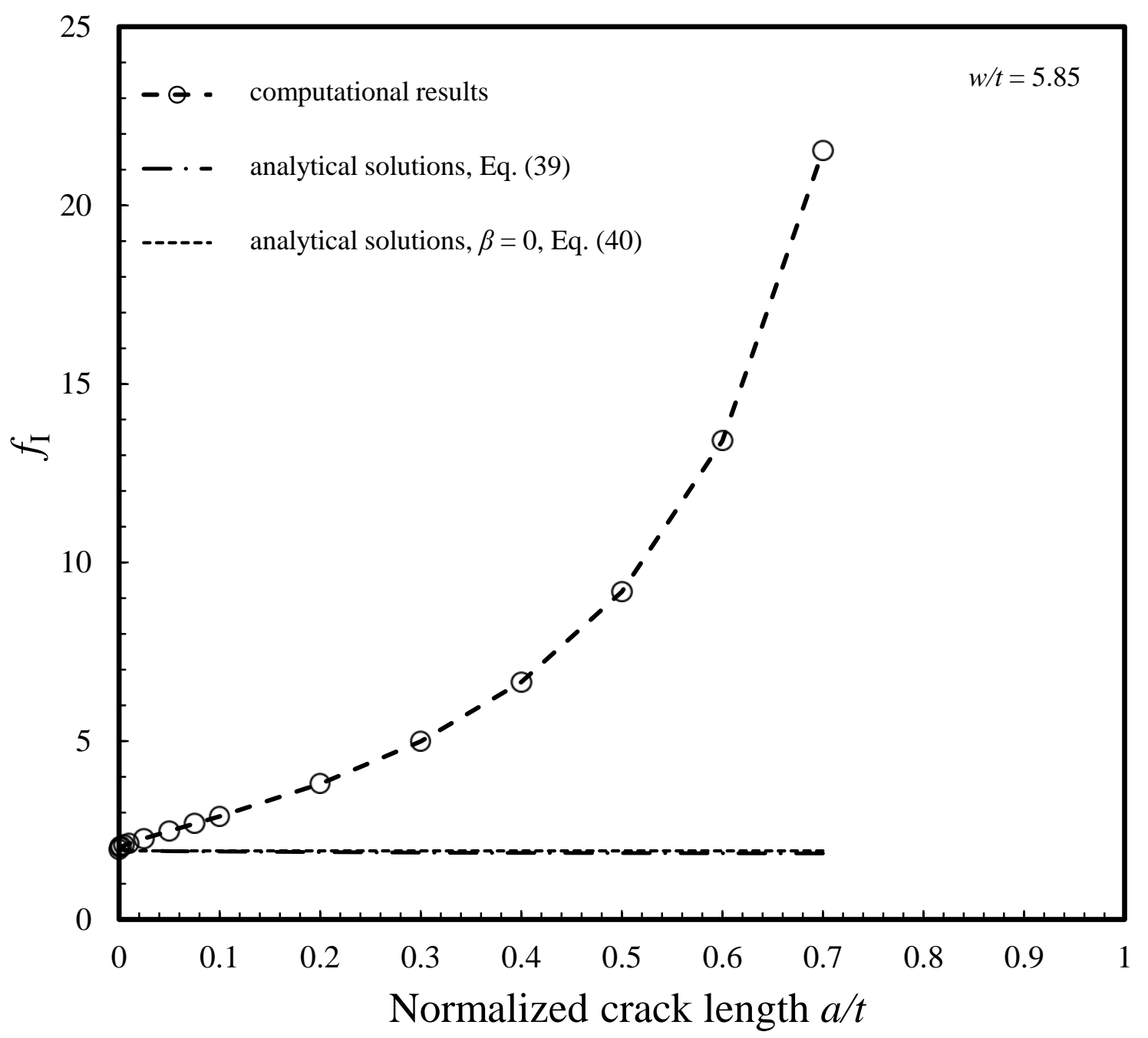

(a) 


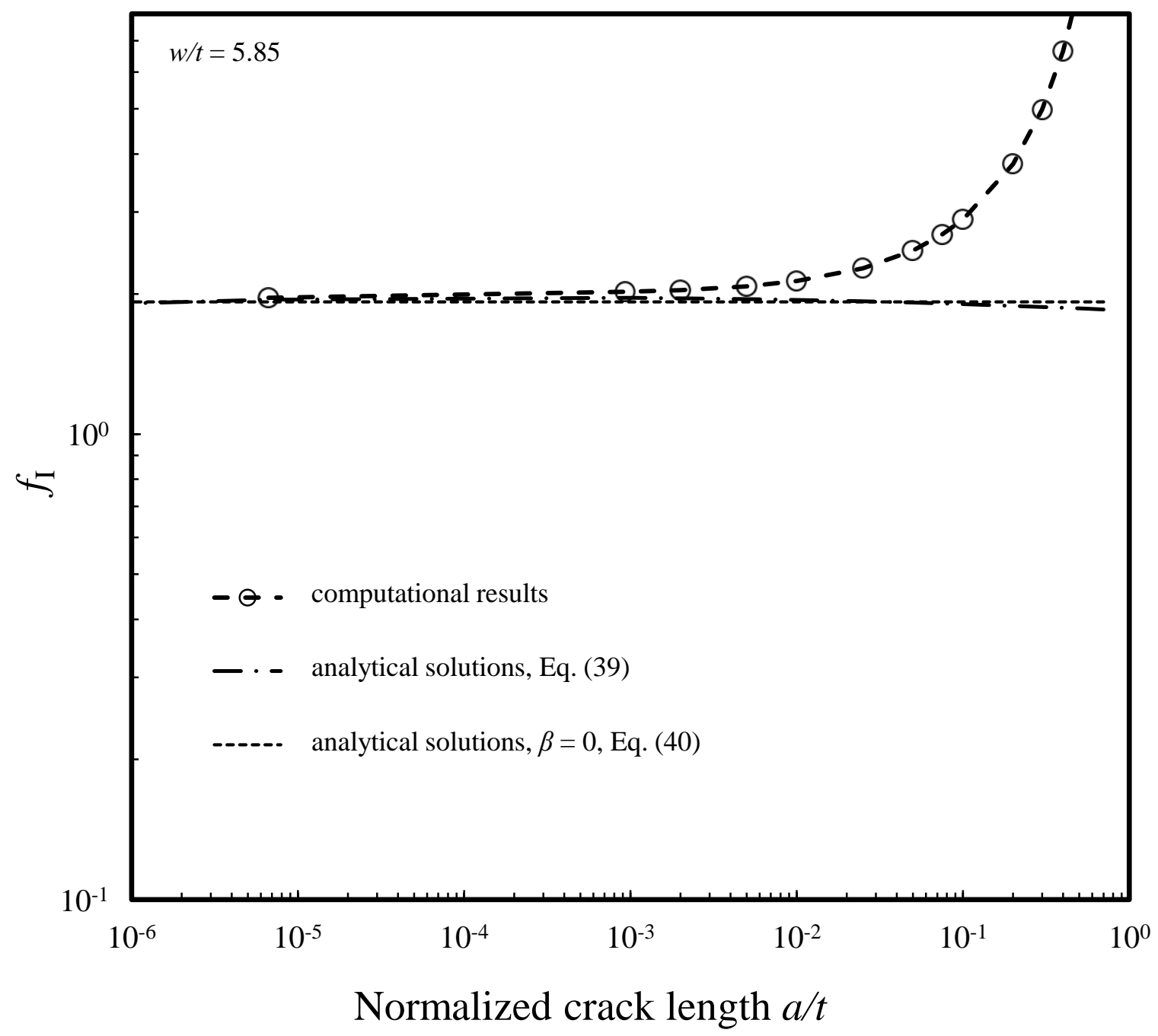

(b) 


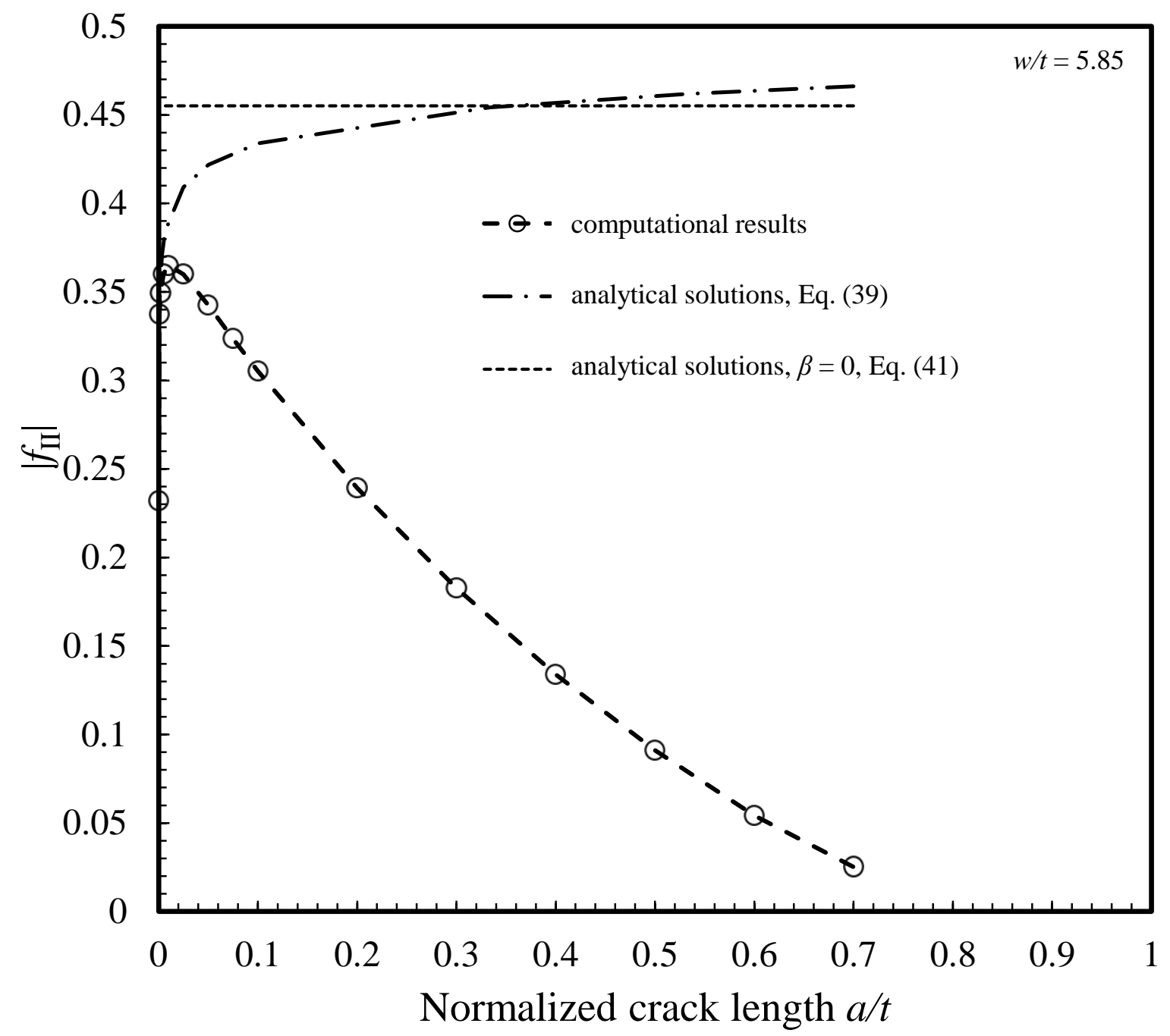

(c) 


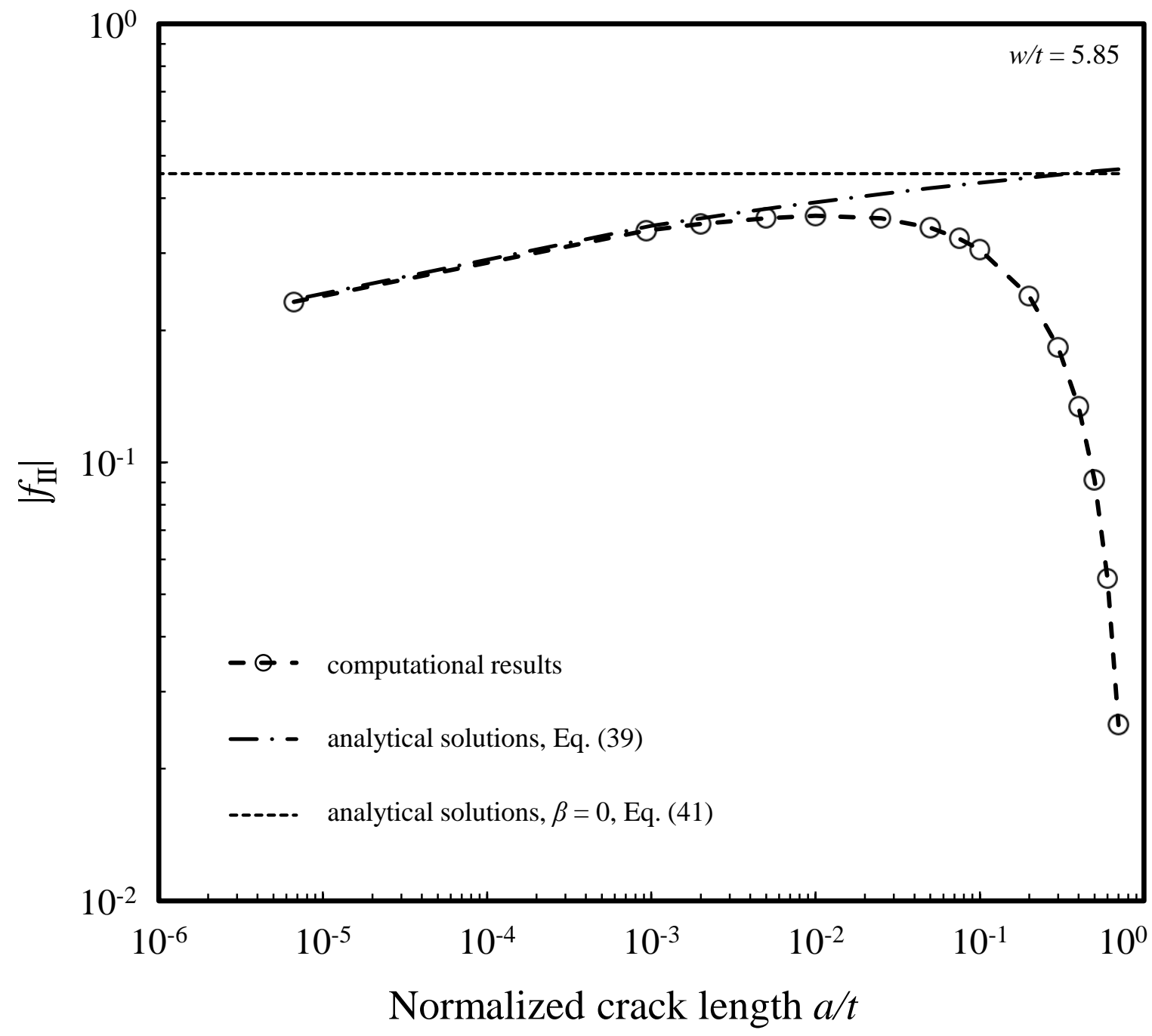

(d) 


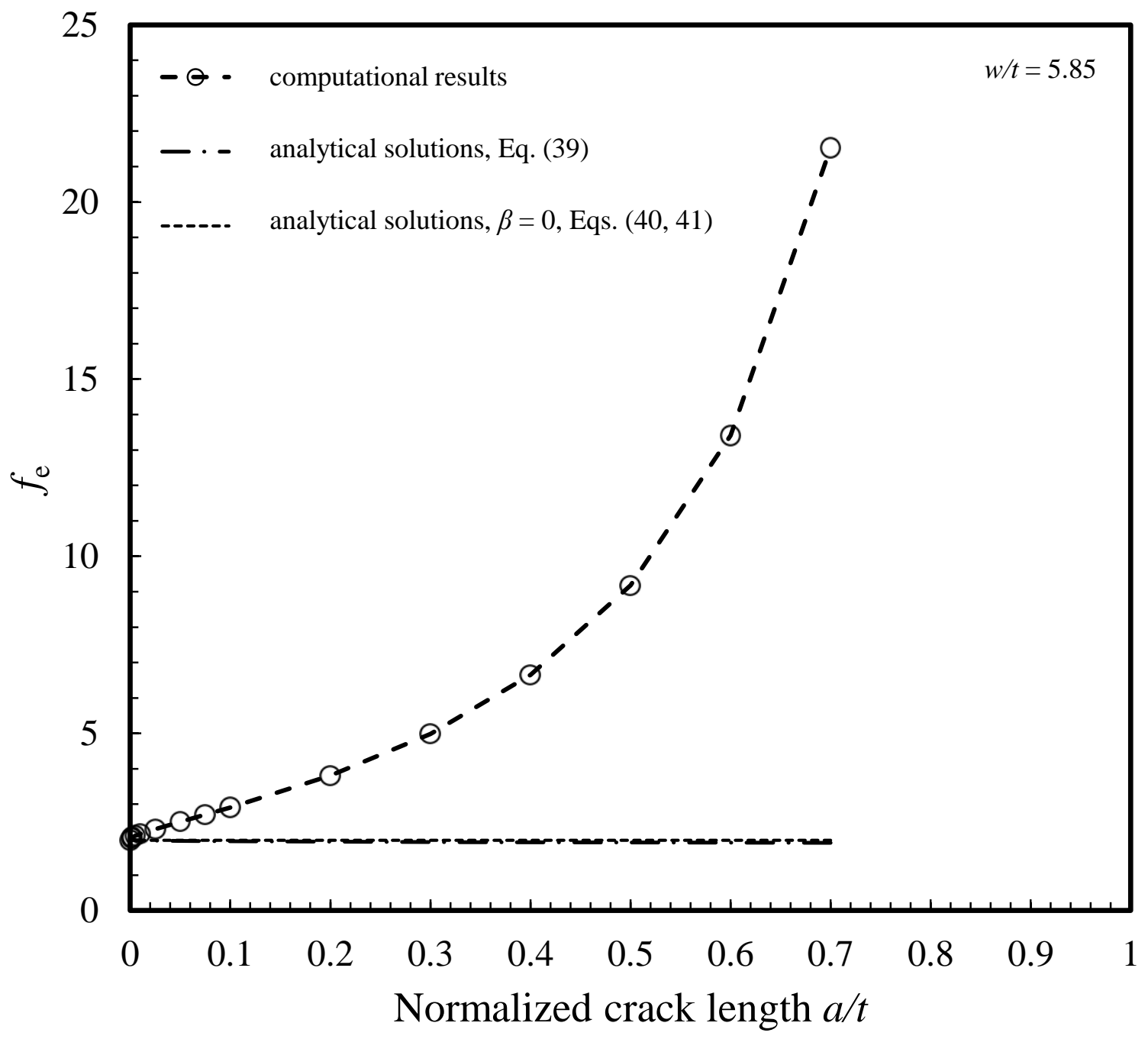

(e) 


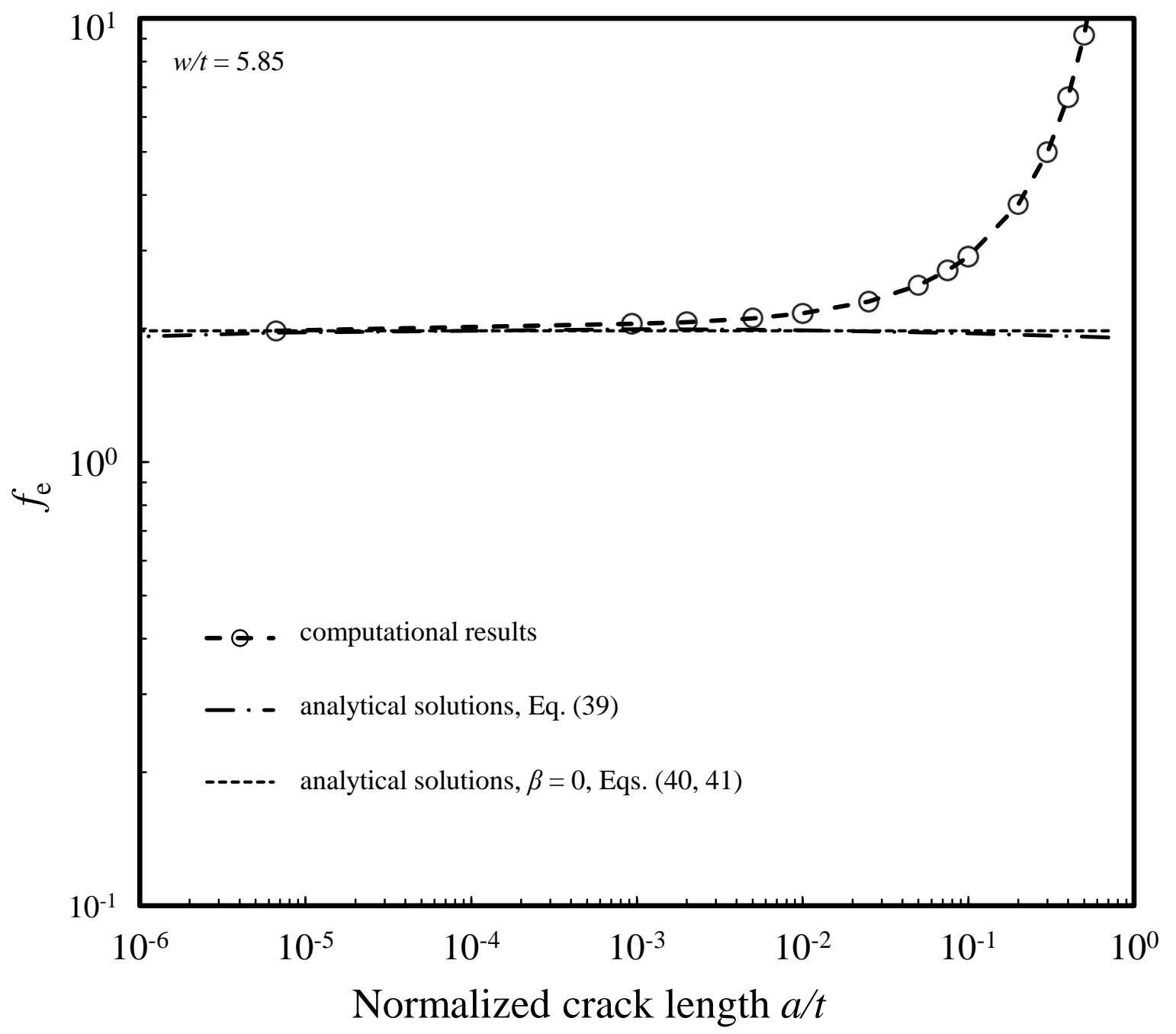

(f)

Figure 11. The computational results and analytical solutions for (a) $f_{\mathrm{I}}$ in the linear scale, (b) $f_{\mathrm{I}}$ in the logarithmic scale, (c) $\left|f_{\mathrm{II}}\right|$ in the linear scale, (d) $\left|f_{\mathrm{II}}\right|$, in the logarithmic scale, (e) $f_{e}$ in the linear scale, and (f) $f_{e}$ in the logarithmic scale, as functions of the normalized kink length $a / t$ for $w / t=5.85$ and $\varphi=-90^{\circ}$.

Figures 11(c) and 11(d) show the computational results and analytical solutions for $\left|f_{\mathrm{II}}\right|$ as functions of the normalized kink length $a / t$ based on both sets of elastic constants in linear and logarithmic scales, respectively. First, it should be noted that the magnitude of the local stress intensity factor $k_{I I}$ is much less than that of the local stress intensity factor $k_{I}$. As expected 
from the analysis of the shear stress directly above the main crack tip in Figure 8(d), the analytical kinked crack solution based on the actual elastic constants in Equation (39) and the modified elastic constants in Equation (41) do not converge as kink length $a$ goes to zero. The analytical kinked crack solution based on the actual elastic constants using the global stress intensity factor solutions and Equation (39) correlates well with the computational results. In Figure 11(d), the analytical solution and the computational results based on the actual elastic constants both decrease as the kink length decreases. The analytical kinked crack solution, however, decreases continually with smaller kink lengths and does not reach a limit. This is a barrier to fatigue prediction as a kinked crack growth model requires a local stress intensity factor evaluated at a crack length of zero or near zero. The analytical kinked crack solution based on the modified elastic constants does not correlate well to the computational results based on the actual elastic constants, however, the magnitude of the results are similar. Equation (41) is only an approximation for the analytical solution based on the actual elastic constants.

However, the $k_{I I}$ solution may not be important for a fatigue crack growth model, where the magnitude of the $k_{I I}$ solution is much less than that of the $k_{I}$ solution.

Figures 11(e) and 11(f) show the computational results for $f_{e}$ as functions of the normalized kink length $a / t$ based on the actual elastic constants in linear and logarithmic scales, respectively. Also shown are the analytical solutions from Equations (39), (40) and (41) based on the actual and modified elastic constants. These figures are remarkably similar to Figures 11(a) and 11(b) for $f_{\mathrm{I}}$. It can be seen from Figure 11(f) that the effective stress intensity factor solution for the vanishing kink length in Equations (40) and (41) based on the modified elastic constants agree with the computational results based on the actual elastic constants as the kink length decreases. This is quite useful because a crack growth model requires the effective stress 
intensity factor $k_{e}$ for the kink length equal to zero or near zero. Therefore, the analytical solution based on the modified elastic constants can be used to obtain the local stress intensity factor solutions for the kink length equal to zero or near zero without impacting the accuracy of the fatigue crack growth model.

Figures 12(a) and 12(b) show the computational results for $f_{\mathrm{I}}$ as functions of the normalized kink length $a / t$ for $w / t=0.5,1$ and 5.85 for the normalized kink length $a / t$ up to 0.7 and for small normalized kink length up to 0.1 , respectively. Figure 12(b) also shows the geometric function of the local stress intensity factor for the vanishing kinked crack, displayed as symbols at $a / t=0$ which were obtained from Equation (40) and the computational $K_{1}$ and $K_{2}$ solutions for the main crack based on the modified elastic constants. Figure 12(b) shows that, as the normalized kink length approaches to 0 , the value of $f_{\mathrm{I}}$ appears to trend toward the local stress intensity factor solutions for the vanishing crack. As shown in these figures, as the normalized kink length increases, the value of $f_{\mathrm{I}}$ increases monotonically. 


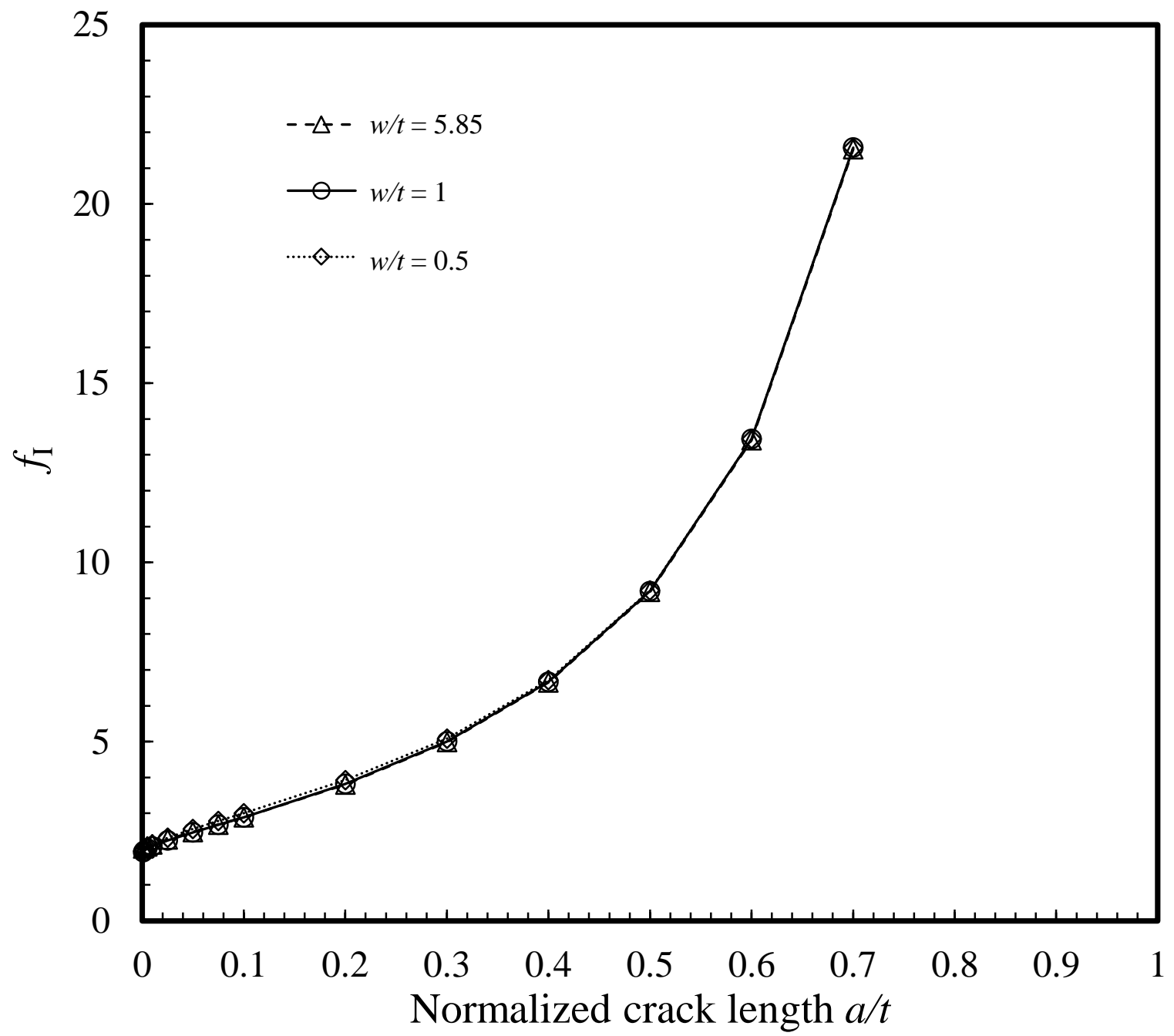

(a) 


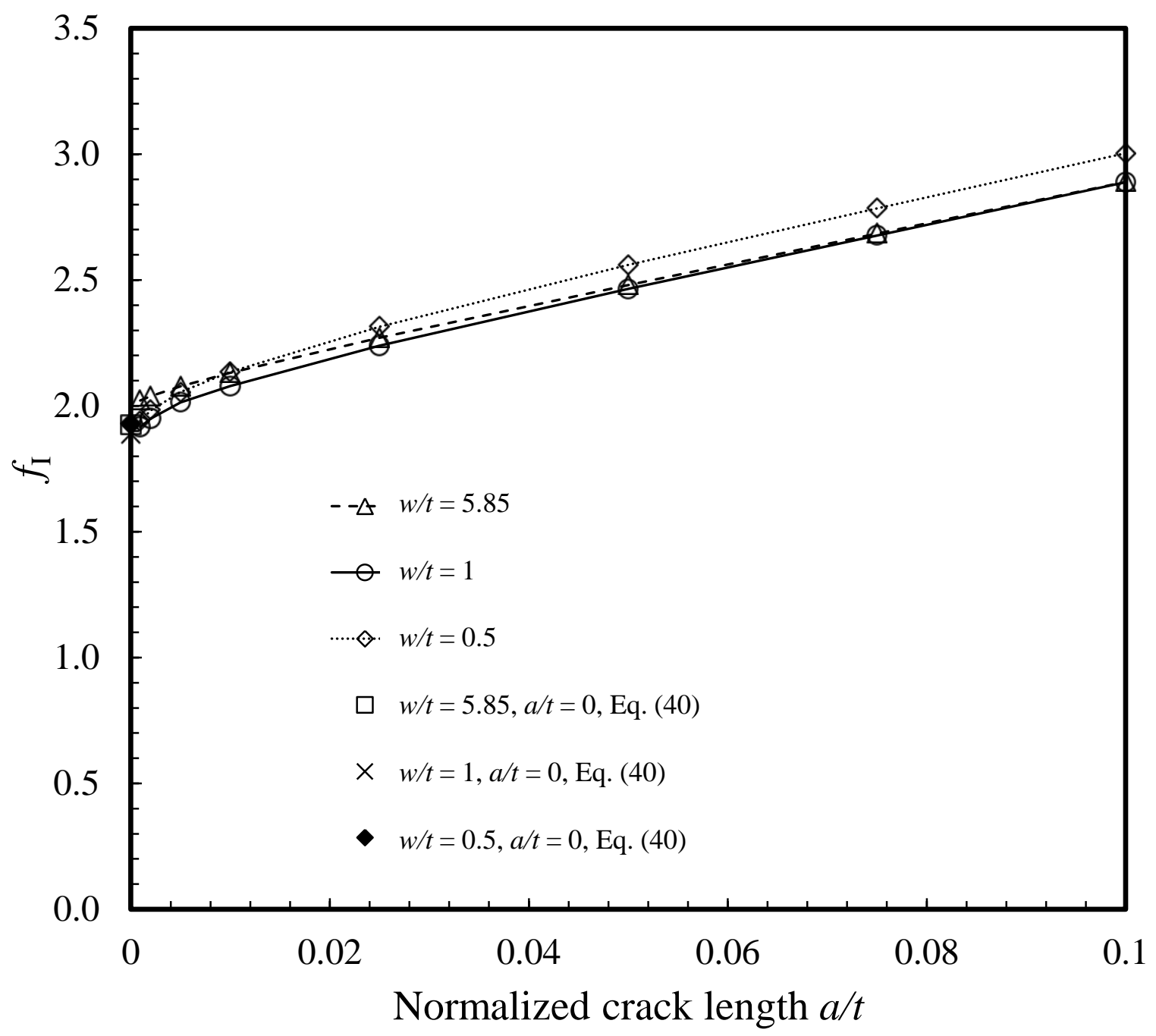

(b)

Figure 12. The computational results for $f_{\mathrm{I}}$ as functions of the normalized kink length $a / t$ for $w / t=0.5,1$ and 5.85 and $\varphi=-90^{\circ}$ for the normalized kink length $a / t$ up to (a) 0.7 and (b) 0.1 .

The computational results for $\left|f_{\mathrm{II}}\right|$ are shown in Figure 13 as functions of the normalized kink length $a / t$ for $w / t=0.5,1$ and 5.85. The geometric function $f_{\text {II }}$ is plotted as the absolute value since the local stress intensity factor solutions $k_{\mathrm{II}}$ are negative according to Figure 9 . Figure 13 also shows the geometric function of the local stress intensity factor for the vanishing kinked crack, displayed as symbols at $a / t=0$ which were obtained from Equation (41) and the 
computational $K_{1}$ and $K_{2}$ solutions for the main crack based on the modified elastic constants.

For all normalized widths, $\left|f_{\mathrm{II}}\right|$ does not appear to trend toward the geometric factors of the local stress intensity factor solutions for the vanishing crack as the normalized kink length approaches zero. The computational solutions for $f_{\mathrm{I}}$ and $\left|f_{\mathrm{II}}\right|$ can only show the trends as the local stress intensity factor solutions approach a kinked crack length of zero. It should be noted that the $f_{\text {I }}$ solution which appears to be accurate at very small kink lengths is more dominant than the $\left|f_{\text {II }}\right|$ solution which is not well approximated. As expected for all three normalized widths, $w / t=0.5,1$ and 5.85, $\left|f_{\mathrm{II}}\right|$ decreases and trends toward zero as the normalized kink length approaches to 1 . For $w / t=0.5$, the maximum value for $\left|f_{\mathrm{II}}\right|$ occurs around $a / t=0.2$, while for $w / t=1$ and 5.85, the maximum value occurs around $a / t=0.025$. 


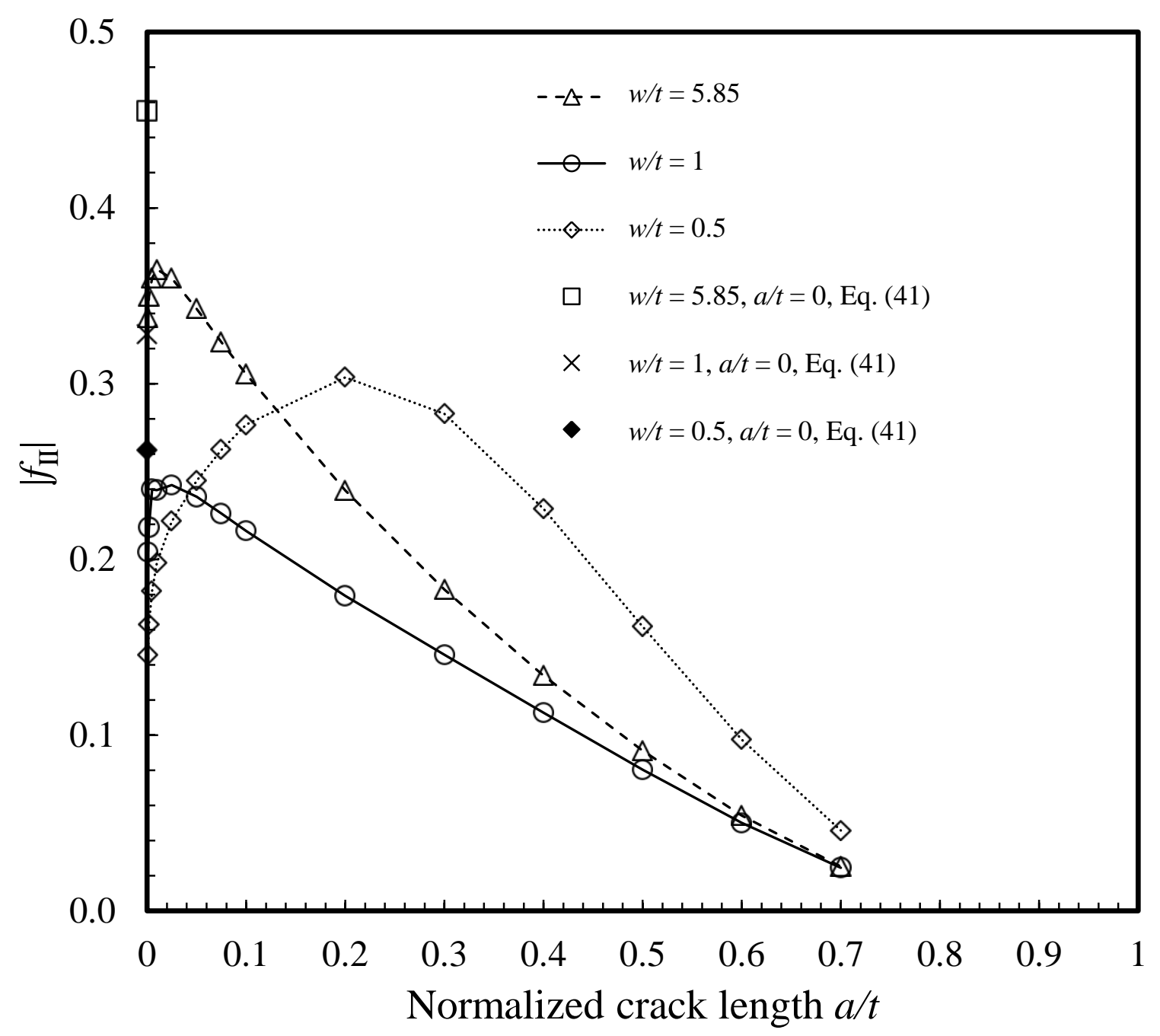

Figure 13. The computational results for $\left|f_{\mathrm{II}}\right|$ as functions of the normalized kink length $a / t$ for $w / t=0.5,1$ and 5.85 and $\varphi=-90^{\circ}$.

Figures 14(a) and 14(b) show the computational results for $f_{e}$ as functions of the normalized kink length $a / t$ for $w / t=0.5,1$ and 5.85, for the normalized kink length up to 0.7 and up to 0.1, respectively. Figure 14(a) appears very similar to Figure 12(a) which shows the computational results for $f_{\mathrm{I}}$. This is consistent with the kinked crack growing under dominant mode I loading conditions. Here, for a kinked crack of zero length, Figure 14(b) shows that the value of $f_{e}$ appears to approach to the local stress intensity factor solutions at $a / t=0$. The 
local stress intensity factors shown as symbols at $a / t=0$, were obtained from Equations (40) and (41) with the computational $K_{1}$ and $K_{2}$ solutions for the main crack for the modified elastic constants. As the value of $a / t$ increases, the value of $f_{e}$ increases monotonically, just as shown in Figure 12(a) for $f_{\mathrm{I}}$.

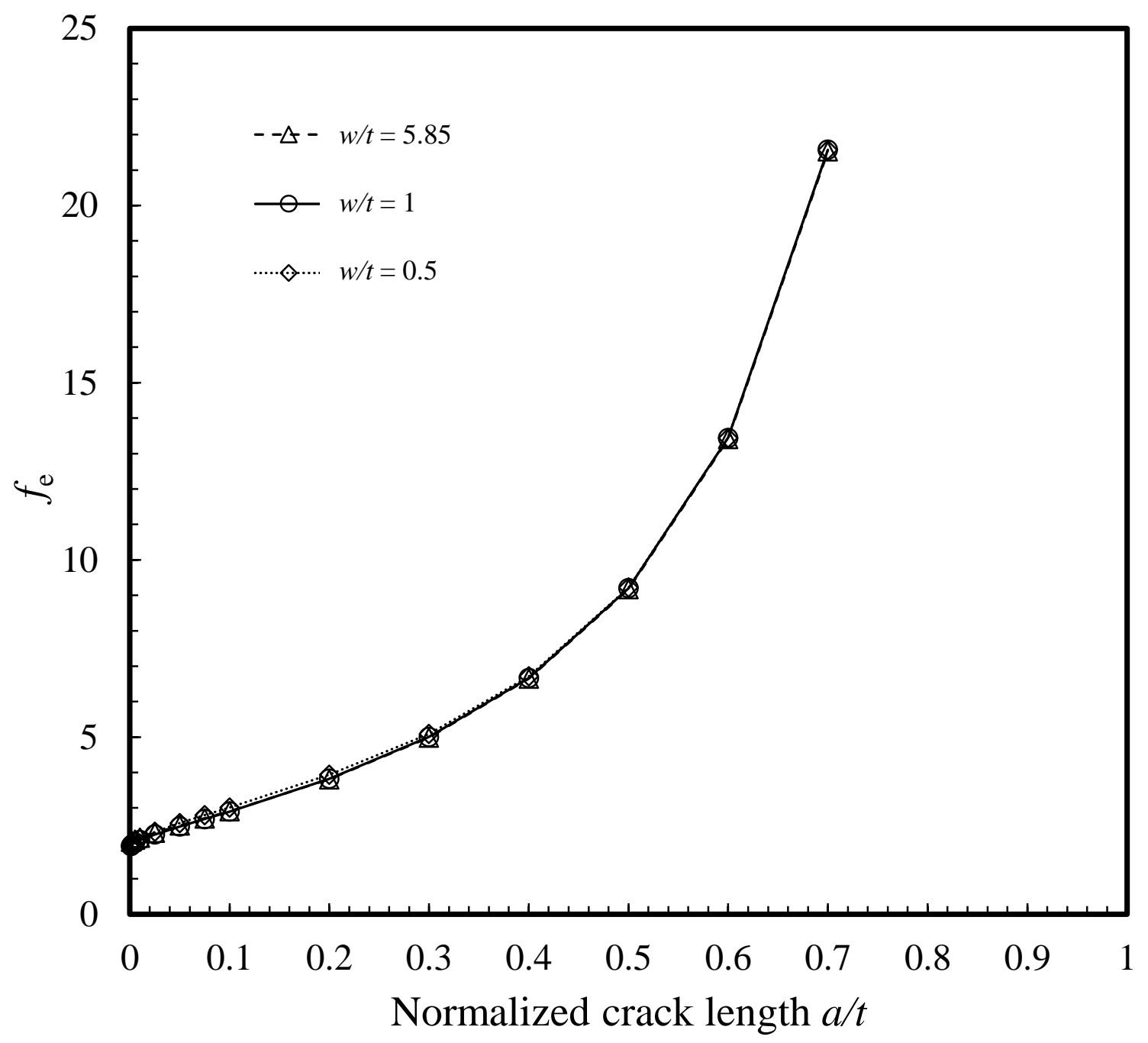

(a) 


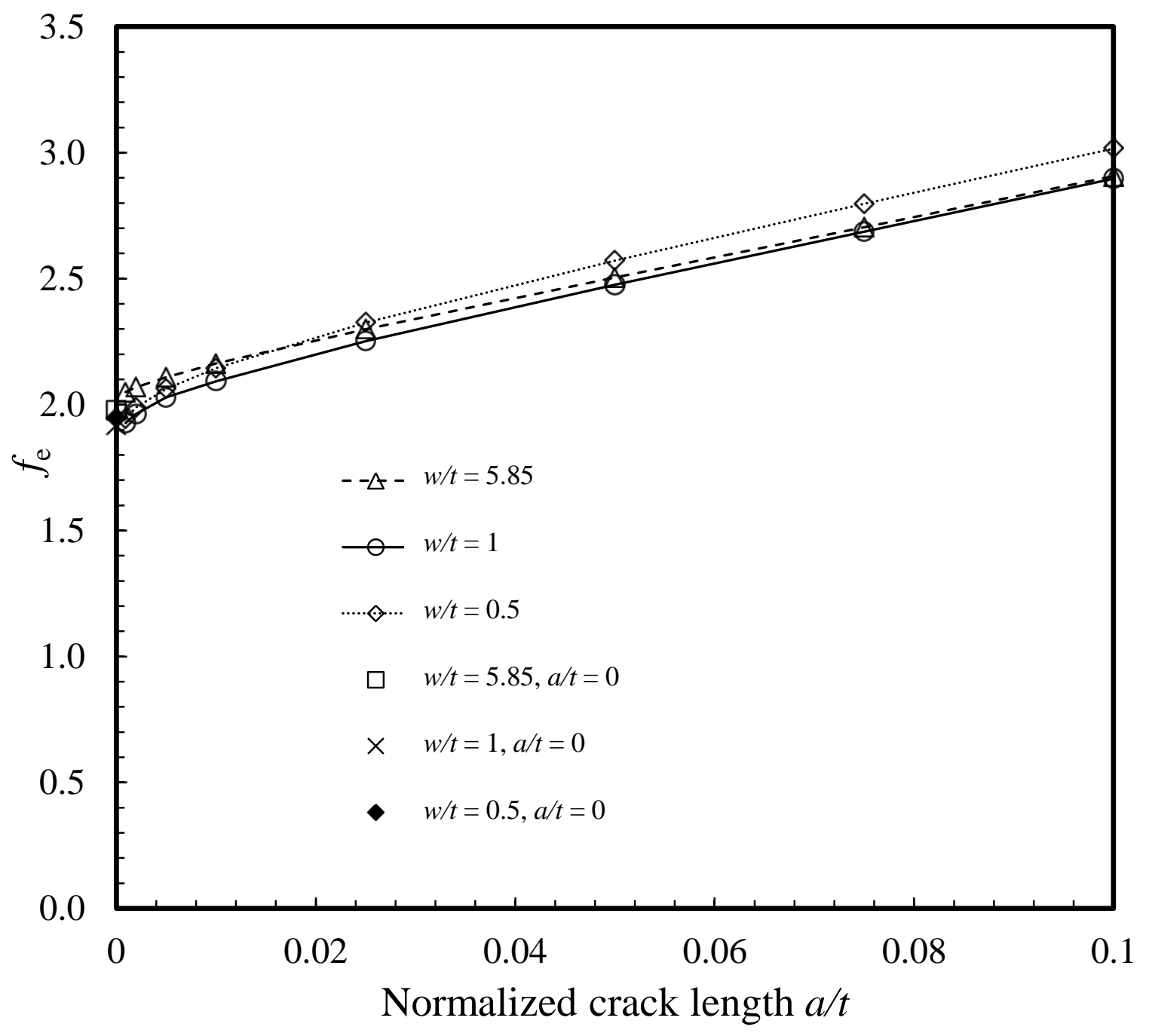




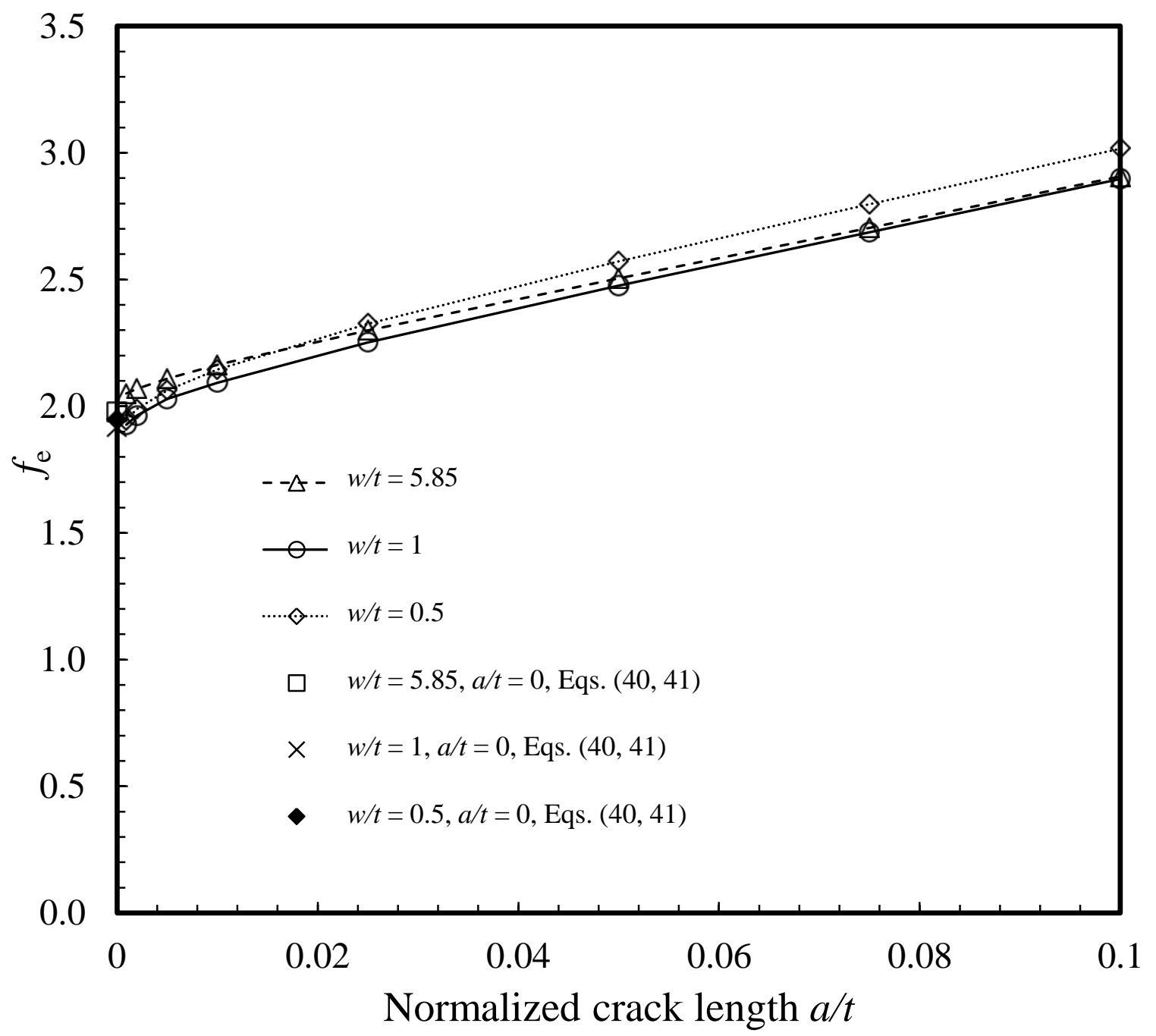

(b)

Figure 14. The computational results for $f_{e}$ as functions of the normalized kink length $a / t$ for $w / t=0.5,1$ and 5.85 and $\varphi=-90^{\circ}$ for the normalized kink length up to (a) 0.7 and (b) 0.1 .

The computational local stress intensity factor $k_{\mathrm{I}}$ solutions trend toward the theoretical vanishing crack solution at $a / t=0$ based on the modified elastic constants as the normalized kink length decreases. The $k_{\text {II }}$ solutions for kinked cracks from the finite element analyses do not approach to the theoretical kinked crack solution at $a / t=0$ based on the modified elastic constants as the normalized kink length decreases. The kinked crack in the welded lap-shear specimen is subjected to stresses from both mode I and mode II. The finite element analyses 
indicate that mode I is more dominant and becomes more pronounced as the kinked crack grows. Therefore the computational $k_{e}$ solutions approach to that for the kinked crack solution at $a / t=0$ based on the modified elastic constants. Tables 5 and 6 list computational results for the dimensionless geometric functions $f_{\mathrm{I}}$ and $\left|f_{\mathrm{II}}\right|$, respectively, for $\varphi=-90^{\circ}, w / t=0.5,1$ and 5.85 based on the actual elastic constants for future engineering applications.

Table 5: The computational results for $f_{\mathrm{I}}$ for $\varphi=-90^{\circ}, w / t=0.5,1$, and 5.85 based on the actual elastic constants.

\begin{tabular}{ccccccccc}
\hline & $\mathrm{a} / \mathrm{t}$ & 0.00093 & 0.002 & 0.005 & 0.01 & 0.025 & 0.05 & 0.075 \\
\hline$f_{\mathrm{I}}$ & $w / t=0.5$ & 1.940 & 1.983 & 2.055 & 2.135 & 2.315 & 2.560 & 2.785 \\
$f_{\mathrm{I}}$ & $w / t=1$ & 1.917 & 1.950 & 2.014 & 2.079 & 2.239 & 2.464 & 2.677 \\
$f_{\mathrm{I}}$ & $w / t=5.85$ & 2.022 & 2.039 & 2.078 & 2.132 & 2.271 & 2.481 & 2.684 \\
\hline
\end{tabular}

Table 5 continued

\begin{tabular}{ccccccccc}
\hline & $\mathrm{a} / \mathrm{t}$ & 0.1 & 0.2 & 0.3 & 0.4 & 0.5 & 0.6 & 0.7 \\
\hline$f_{\mathrm{I}}$ & $w / t=0.5$ & 3.003 & 3.927 & 5.081 & 6.712 & 9.218 & 13.443 & 21.569 \\
$f_{\mathrm{I}}$ & $w / t=1$ & 2.889 & 3.820 & 5.004 & 6.669 & 9.202 & 13.443 & 21.573 \\
$f_{\mathrm{I}}$ & $w / t=5.85$ & 2.890 & 3.802 & 4.985 & 6.646 & 9.175 & 13.408 & 21.526 \\
\hline
\end{tabular}

Table 6: The computational results for $\left|f_{\text {II }}\right|$ for $\varphi=-90^{\circ}, w / t=0.5,1$, and 5.85 based on the actual elastic constants.

\begin{tabular}{ccccccccc}
\hline & $\mathrm{a} / \mathrm{t}$ & 0.00093 & 0.002 & 0.005 & 0.01 & 0.025 & 0.05 & 0.075 \\
\hline$\left|f_{\mathrm{II}}\right|$ & $w / t=0.5$ & 0.146 & 0.163 & 0.182 & 0.198 & 0.222 & 0.245 & 0.262 \\
$\left|f_{\mathrm{II}}\right|$ & $w / t=1$ & 0.204 & 0.218 & 0.240 & 0.239 & 0.242 & 0.236 & 0.226 \\
$\left|f_{\mathrm{II}}\right|$ & $w / t=5.85$ & 0.337 & 0.350 & 0.360 & 0.365 & 0.360 & 0.343 & 0.324 \\
\hline
\end{tabular}

Table 6 continued

\begin{tabular}{ccccccccc}
\hline & $\mathrm{a} / \mathrm{t}$ & 0.1 & 0.2 & 0.3 & 0.4 & 0.5 & 0.6 & 0.7 \\
\hline$\left|f_{\mathrm{II}}\right|$ & $w / t=0.5$ & 0.277 & 0.304 & 0.283 & 0.229 & 0.162 & 0.097 & 0.046 \\
$\left|f_{\mathrm{II}}\right|$ & $w / t=1$ & 0.216 & 0.179 & 0.146 & 0.113 & 0.080 & 0.050 & 0.025 \\
$\left|f_{\mathrm{II}}\right|$ & $w / t=5.85$ & 0.305 & 0.239 & 0.183 & 0.134 & 0.091 & 0.054 & 0.025 \\
\hline
\end{tabular}




\section{Conclusions}

Stress intensity factor solutions for welds with various widths in lap-shear specimens of magnesium alloy AZ31 and HSLA steel sheets with and without kinked cracks are developed using finite element analyses. First, the analytical stress intensity factor solutions for lap-shear specimens based on the beam bending theory and the analytical solutions for two dissimilar semi-infinite planes with connection under plane strain conditions are reviewed. Finite element analyses of lap-shear specimens were conducted with multiple weld widths using the actual material properties for both magnesium and steel sheets to obtain the global stress intensity factor solutions for main cracks. The results of the finite element analyses are compared with the analytical stress intensity factor solutions based on the beam bending theory and for semi-infinite planes with connection to identify the transition widths and to validate the finite element analyses. Finite element analyses were also conducted for the lap-shear specimens of the magnesium and steel sheets, but with modified elastic constants for the magnesium sheet such that the bimaterial constant is equal to zero. The results indicate that the crack-tip stresses directly ahead of the main crack tip are influenced by the oscillation of the interface crack-tip field at a very small distance to the tip. However, the crack-tip stresses directly above the main crack tips are only weakly affected, even at a very small distance to the tip, and the oscillatory crack-tip stress distributions based on both actual and modified elastic constants are quite similar. The results indicate that the stress intensity factor solutions for kinked crack with the vanishing kink length can be approximated by the available analytical solutions for the zero bimaterial constant for fatigue life estimations. Finite element analyses were also performed for lap-shear specimens with multiple weld widths to obtain the local stress intensity factor solutions 
for kinked cracks with the experimentally observed kink angle as functions of the kink length. The stress intensity factor solutions for the main and kinked crack can be used along with the solutions for the vanishing crack, with modified elastic constants, for fatigue life estimations of ultrasonic welds in lap-shear specimens of magnesium and steel sheets. 


\section{Acknowledgements}

This research was initially sponsored by the U.S. Department of Energy, Assistant

Secretary for Energy Efficiency and Renewable Energy, Office of Vehicle Technologies, as part

of the Lightweight Materials Program. The support of this work through a National Science

Foundation Fellowship for TR is greatly appreciated.

\section{References}

[1] Lang B, Sun DQ, Xuan ZZ, Qin XF. Hot cracking of resistance spot welded magnesium alloy. ISIJ Int 2008;48(1):77-82.

[2] Patel VK, Bhole SD, Chen DL. Ultrasonic spot welded AZ31 magnesium alloy: microstructure, texture, and lap shear strength. Mater Sci Eng, A 2013;569:78-85.

[3] Hetrick E, Jahn R, Reatherford L, Skogsmo J, Ward SM, Wilkosz D, et al. Ultrasonic spot welding: A new tool for aluminum joining. Weld J 2005;84(2):26-30.

[4] Jahn R, Cooper R, Wilkosz D. The effect of anvil geometry and welding energy on microstructures in ultrasonic spot welds of AA6111-T4. Metall Mater Trans A 2007;38A:570-83.

[5] Bakavos D, Prangnell PB. Mechanisms of joint and microstructure formation in high power ultrasonic spot welding 6111 aluminium automotive sheet. Mater Sci Eng, A 2010;527:632034.

[6] Jordan JB, Horstemeyer MF, Daniewicz SR, Badarinarayan H, Grantham J. Fatigue characterization and modeling of friction stir spot welds in magnesium AZ31 alloy. $\mathrm{J}$ Eng Mater Technol 2010;132:041008-1-10.

[7] Gendo T, Nishiguchi K, Asakawa M, Tanioka S. Spot friction welding of aluminum to steel. SAE Technical Paper 2007-01-1703, Society of Automotive Engineers, Warrendale, PA.

[8] Liyanage T, Kilbourne J, Gerlich AP, North TH. Joint formation in dissimilar Al alloy/steel and Mg alloy/steel friction stir spot welds. Sci Technol Weld Joining 2009;14:500-8.

[9] Watanabe T, Sakuyama H, Yanagisawa A. Ultrasonic welding between mild steel sheet and Al-Mg alloy sheet. J Mater Process Technol 2009;209(15-16):5475-80.

[10] Santella M, Franklin T, Pan J, Pan T-Y, Brown E. Ultrasonic spot welding of AZ31B to galvanized mild steel. SAE Technical Paper 2010-01-0975, Society of Automotive Engineers, Warrendale, PA. 
[11] Jana S, Hovanski Y. Fatigue behaviour of magnesium to steel dissimilar friction stir lap joints. Sci Technol Weld Joining 2012;17(2):141-5.

[12] Uematsu Y, Kakiuchi T, Tozaki Y, Kojin H. Comparative study of fatigue behaviour in dissimilar $\mathrm{Al}$ alloy/steel and $\mathrm{Mg}$ alloy/steel friction stir spot welds fabricated by scroll grooved tool without probe. Sci Technol Weld Joining 2012;17(5):348-56.

[13] Patel VK, Bhole SD, Chen DL. Fatigue life estimation of ultrasonic spot welded Mg alloy joints. Mater Des 2014;62:124-132.

[14] Shen Z, Ding Y, Chen J, Gerlich AP. Comparison of fatigue behavior in $\mathrm{Mg} / \mathrm{Mg}$ similar and $\mathrm{Mg} /$ steel dissimilar refill friction stir spot welds. Int J Fatigue 2016;92:78-86.

[15] Ueoka T, Tsujino J. Welding characteristics of aluminum and copper plate specimens welded by a $19 \mathrm{kHz}$ complex vibration ultrasonic seam welding system. Jpn J Appl Phys 2002;41:3237-42.

[16] Sripichai K, Asim K, Pan J. Stress intensity factor solutions for estimation of fatigue lives of laser welds in lap-shear specimens. Eng Fract Mech 2011;78:1424-40.

[17] Zhang S. Stress intensities at spot welds. Int J Fract 1997;88:167-85.

[18] Zhang S. Fracture mechanics solutions to spot welds. Int J Fract 2001;112:247-75.

[19] Suo Z, Hutchinson JW. Interface crack between two elastic layers. Int J Fract 1990;43:1-18.

[20] Lee J, Asim K, Pan J. Modeling of failure mode of laser welds in lap-shear specimens of HSLA steel sheets. Eng Fract Mech 2011;78:374-96.

[21] Asim K, Lee J, Pan J. Failure mode of laser welds in lap-shear specimens of high strength low alloy (HSLA) steel sheets. Fatigue Fract Eng Mater Struct 2011;35:219-237.

[22] Anand D, Chen DL, Bhole SD, Andreychuk P, Boudreau G. Fatigue behavior of tailor (laser)-welded blanks for automotive applications. Mater Sci Eng, A 2006;420:199-207.

[23] Asim K, Sripichai K, Pan J. Fatigue behavior of laser welds in lap-shear specimens of high strength low alloy (HSLA) steel sheets. Int J Fatigue 2014;61:283-296.

[24] He M-Y, Hutchinson JW. Kinking of a crack out of an interface. J Appl Mech 1989;56(2):270-8.

[25] Erdogan F. Stress distribution in a nonhomogeneous elastic plane with cracks. J Appl Mech 1963;30(2):232-6.

[26] Rice JR, Sih GC. Plane problems of cracks in dissimilar media. J Appl Mech 1965;32:41823.

[27] Rice JR. Elastic fracture mechanics concepts for interfacial cracks. J Appl Mech 1988;55:98-103. 
[28] Zhang S. Stress intensities derived from stresses around a spot weld. Int J Fract 1999;99:239-257.

[29] Tada H, Paris PC, Irwin GR. The stress analysis of cracks handbook. $3^{\text {rd }}$ edition. New York: ASME Press; 2000.

[30] Lee J. Simulations of similar and dissimilar weld joint failures and bearings for fatigue analyses [doctoral dissertation]. Ann Arbor, MI: University of Michigan; 2011.

[31] Franklin T. Fatigue Behavior of Dissimilar Ultrasonic Welds in Lap-Shear Specimens of AZ31 and Steel Sheets. Ann Arbor, MI: University of Michigan; 2011.

[32] ABAQUS v6.10 User Manual. Providence, RI: SIMULIA; 2010.

[33] He M-Y, Hutchinson JW. Kinking of a crack out of an interface: tabulated solution coefficients. Harvard University Report MECH-113A. 1988.

[34] Cotterell B, Rice JR. Slightly curved or kinked cracks. Int J Fract 1980;16:155-69. 


\section{Figures captions}

Figure 1. (a) A top view and (b) a bottom view of an ultrasonic welded lap-shear specimen which has been machined into a dog-bone shape. (c) A schematic of a lap-shear specimen. The applied force $F$ is shown as the bold arrows.

Figure 2. An optical micrograph of a failed weld at the fatigue life of $1.7 \times 10^{3}$ cycles under a load range of $1.70 \mathrm{kN}$.

Figure 3. (a) A schematic of the weld with the lap-shear load. (b) A schematic of the left crack tip showing the normal stresses $\sigma_{u i}, \sigma_{u o}, \sigma_{l i}$ and $\sigma_{l o}$ at the inner $(i)$ and outer $(o)$ surfaces of the upper $(u)$ and lower $(l)$ strips, respectively.

Figure 4. A schematic diagram of two half planes of dissimilar materials with connection of the length $w$. The Cartesian $x-y$ coordinate system is shown. The shear forces per unit width, $F / b$, are applied along the $x$ axis at $x=+\infty$ and $-\infty$ of the upper and lower planes, respectively. The normal forces per unit width, $N / b$, are applied along the $y$ axis at $y=+\infty$ and $-\infty$ of the upper and lower planes, respectively.

Figure 5. (a) A schematic of a two-dimensional finite element model of the lap-shear specimen with the boundary and loading conditions. (b) A view of the finite element mesh for the central portion of the finite element mesh and (c) a close-up view of the finite element mesh near the right crack tip for $w / t=5.85$.

Figure 6. The normalized effective stress intensity factor solutions as functions of $w / t$ plotted in (a) linear and (b) logarithmic scales.

Figure 7. The finite element mesh near the right main crack indicating the directions ahead of the crack tip $\theta=0^{\circ}$, and above the crack tip $\theta=-90^{\circ}$, as pointing horizontally and vertically from the main crack tip, respectively.

Figure 8. A comparison of (a) the normal stress in the $y$ direction ahead of the crack tip, (b) the shear stress ahead of the crack tip, (c) the normal stress in the $x$ direction vertically above the crack tip, and (d) the shear stress vertically above the crack tip from the finite element analyses based on the actual elastic constants and the modified elastic constants.

Figure 9. A schematic of a main crack and a kinked crack with the kink length $a$ and the kink angle $\varphi$.

Figure 10. (a) A schematic of a two-dimensional finite elemental model of a lap-shear specimen with a kinked crack with the boundary and loading conditions. (b) The finite element mesh showing the pre-existing cracks and the kinked crack on the right hand side for $w / t=5.85$ and $a / t=0.1$, (c) the finite element mesh near the right main crack tip, and (d) a close-up view of the finite element mesh near the kinked crack tip. 
Figure 11. The computational results and analytical solutions for (a) $f_{\mathrm{I}}$ in the linear scale, (b) $f_{\mathrm{I}}$ in the logarithmic scale, (c) $\left|f_{\mathrm{II}}\right|$ in the linear scale, (d) $\left|f_{\mathrm{II}}\right|$, in the logarithmic scale, (e) $f_{e}$ in the linear scale, and (f) $f_{e}$ in the logarithmic scale, as functions of the normalized kink length $a / t$ for $w / t=5.85$ and $\varphi=-90^{\circ}$.

Figure 12. The computational results for $f_{\mathrm{I}}$ as functions of the normalized kink length $a / t$ for $w / t=0.5,1$ and 5.85 and $\varphi=-90^{\circ}$ for the normalized kink length $a / t$ up to (a) 0.7 and (b) 0.1 .

Figure 13. The computational results for $\left|f_{\mathrm{II}}\right|$ as functions of the normalized kink length $a / t$ for $w / t=0.5,1$ and 5.85 and $\varphi=-90^{\circ}$.

Figure 14. The computational results for $f_{e}$ as functions of the normalized kink length $a / t$ for $w / t=0.5,1$ and 5.85 and $\varphi=-90^{\circ}$ for the normalized kink length up to (a) 0.7 and (b) 0.1 . 